Subscriber access provided by Lancaster University Library

\title{
3D Protein-Based Bilayer Artificial Skin for Guided Scarless Healing of 3rd degree Burn Wounds In vivo
}

Mazaher Gholipourmalekabadi, Alexander M. Seifalian, Aleksandra M. Urbanska, Mir Davood Omrani, John G. Hardy, Zahra Madjd, Seyed Mahmoud Hashemi, Hossein Ghanbarian, Peiman

Brouki Milan, Masoud Mozafari, Rui L. Reis, Subhas C. Kundu, and Ali Samadikuchaksaraei

Biomacromolecules, Just Accepted Manuscript • DOI: 10.1021/acs.biomac.7b01807 • Publication Date (Web): 12 Mar 2018

Downloaded from http://pubs.acs.org on March 19, 2018

\section{Just Accepted}

"Just Accepted" manuscripts have been peer-reviewed and accepted for publication. They are posted online prior to technical editing, formatting for publication and author proofing. The American Chemical Society provides "Just Accepted" as a service to the research community to expedite the dissemination of scientific material as soon as possible after acceptance. "Just Accepted" manuscripts appear in full in PDF format accompanied by an HTML abstract. "Just Accepted" manuscripts have been fully peer reviewed, but should not be considered the official version of record. They are citable by the Digital Object Identifier (DOI®). "Just Accepted" is an optional service offered to authors. Therefore, the "Just Accepted" Web site may not include all articles that will be published in the journal. After a manuscript is technically edited and formatted, it will be removed from the "Just Accepted" Web site and published as an ASAP article. Note that technical editing may introduce minor changes to the manuscript text and/or graphics which could affect content, and all legal disclaimers and ethical guidelines that apply to the journal pertain. ACS cannot be held responsible for errors or consequences arising from the use of information contained in these "Just Accepted" manuscripts. 


\section{D Protein-Based Bilayer Artificial Skin for Guided Scarless Healing of $3^{\text {rd }}$ degree Burn Wounds In vivo}

Mazaher Gholipourmalekabadi ${ }^{1,2, *}$, Alexander M. Seifalian ${ }^{3}$, Aleksandra M. Urbanska ${ }^{4}$, Mir Davood Omrani ${ }^{5}$, John G. Hardy ${ }^{6,7}$, Zahra Madjd ${ }^{8}$, Seyed Mahmoud Hashemi ${ }^{9}$, Hossein Ghanbarian $^{10,11, *}$, Peiman Brouki Milan ${ }^{1,2}$, Masoud Mozafari ${ }^{1,2}$, Rui L. Reis ${ }^{12}$, Subhas C. Kundu ${ }^{12}$, Ali Samadikuchaksaraei ${ }^{1,2}$

${ }^{1}$ Cellular and Molecular Research Centre, Iran University of Medical Sciences, Tehran, Iran

2 Department of Tissue Engineering \& Regenerative Medicine, Faculty of Advanced Technologies in Medicine, Iran University of Medical Sciences, Tehran, Iran

${ }^{3}$ Nanotechnology \& Regenerative Medicine Commercialization Centre (Ltd), The London BioScience Innovation Centre, London, UK

4 Division of Digestive and Liver Disease, Department of Medicine and Herbert Irving Comprehensive Cancer Center, Columbia University, New York, New York 10032, USA

${ }^{5}$ Department of Medical Genetics, Faculty of Medicine, Shahid Beheshti University of Medical Sciences, Tehran, Iran

${ }^{6}$ Department of Chemistry, Lancaster University, Lancaster, Lancashire, United Kingdom

${ }^{7}$ Materials Science Institute, Lancaster University, Lancaster, Lancashire, United Kingdom

${ }^{8}$ Oncopathology Research Center, Iran University of Medical Sciences, Tehran, Iran

${ }^{9}$ Department of Immunology, School of Medicine, Iran University of Medical Sciences, Tehran, Iran

${ }^{10}$ Cellular \& Molecular Biology Research Centre, Shahid Beheshti University of Medical Science, Tehran, Iran

${ }^{11}$ Biotechnology Department, School of Advanced Technologies in Medicine, Shahid Beheshti University of Medical Sciences, Tehran, Iran

12 3Bs Research Group, Headquarters of the European Institute of Excellence on Tissue Engineering and Regenerative Medicine, University of Minho, AvePark - 4805-017 Barco, Guimaraes, Portugal. 


\section{*Corresponding authors:}

Dr. Mazaher Gholipourmalekabadi

Department of Tissue Engineering \& Regenerative Medicine, Iran University of Medical Sciences, Hemmat Highway, Tehran 144961-4535, Iran; Tel: (+98 21) 8862 2755; Fax: (+98 21) 88622533

E-mail: mazaher.gholipour@gmail.com; mazaher.gholipour@sbmu.ac.ir

Dr. Hossein Ghanbarian

Biotechnology Department, School of Advanced Technologies in Medicine, Shahid Beheshti University of Medical Sciences, Koodakyar Street, Velenjak, 987898-6656 Tehran, Iran; Tel: 0098021 22439957; Fax: 009802122439956

E-mail: ghanbarian.hossein@gmail.com; hghanbarian@sbmu.ac.ir 


\begin{abstract}
Severe burn injuries can lead to delay in healing and devastating scar formation. Attempts are made to develop a suitable skin substitute for scarless healing of such skin wounds. Currently, there is no effective strategy yet for a complete scarless healing after the thermal injuries. In our recent work we fabricate and evaluated a 3D protein-based artificial skin made from decellularized human amniotic membrane (AM) and electrospun nanofibrous silk fibroin (ESF) in vitro. We also characterize both biophysical and cell culture investigation to establish in vitro performance of the developed bilayer scaffolds. In this report we evaluate finally about the appropriate utility of this fabricated bi-layered artificial skin in vivo with particular reference to healing and scar formation due to biochemical and biomechanical complexities of the skin. For this work. AM, AM/ESF alone or seeded with adipose tissue-derived mesenchymal stem cells (AT-MSCs) are implanted to full thickness burn wounds in mice. The healing efficacy and scar formation are evaluated at 7, 14 and 28 days post-implantation in vivo. Our data reveal that ESF accelerates wound healing process through early recruitment of inflammatory cells such as macrophages into the defective site, as well as up-regulation of angiogenic factors from the ATMSCs and facilitation of remodeling phase. In vivo application of the prepared AM/ESF membrane seeded with the AT-MSCs reduces significantly the post-burn scars. The in vivo data suggest that the potential applications of the AM/ESF bi-layered artificial skin may be considered as a clinically translational product with stem cells to guide scarless healing of sever burn injuries.
\end{abstract}

Keywords: human amniotic membrane; silk fibroin; electrospinning; artificial skin; wound healing; scar. 


\section{INTRODUCTION}

Burn injuries are among the most devastating of all injuries and present a major global public health crisis ${ }^{1,2}$. Lack of an appropriate burn care strategy and delayed treatment pose a great threat to human health and life ${ }^{3}$. While in high-income countries burn death rates are on a decline, the direct costs for care of children with burns in the United States of America exceeded US\$ 211 million in 2011. Moreover, in low-income countries such as India over 1000000 people are moderately or severely burnt every year ${ }^{4}$. It is well documented that $3^{\text {rd }}$ degree burn wounds require skin grafting to accelerate wound healing and prevent post-burn infections. This subsequently results in scarless healing of the wound ${ }^{5,6}$. Many efforts are made to develop skin substitutes, some of which are already used in clinical practices (SI Table 1). However, the challenges in total skin reconstruction with complete function, especially in $3^{\text {rd }}$ degree burn injuries treatment, remain a major concern at burn intensive care units ${ }^{5,7-9}$. An ideal tissueengineered skin substitute should be biocompatible, biodegradable, impermeable to external bacteria, prevent dehydration, have a degradation rate comparable to the healing process, have a rapid and sustained adherence to the wound surface, have an inner surface structure to allow for cell migration/proliferation/in-growth of new tissue and be flexible and pliable so it can conform to irregular wound surfaces. Moreover, a skin substitute should be readily available, reproducible, cost-effective and require minimal storage requirements ${ }^{7,10,11}$.

In our recent report, we fabricate a $3 \mathrm{D}$ protein-based bi-layered artificial skin made from decellularized human amniotic membrane (AM) and electrospun nanofibrous silk fibroin (ESF). We characterize in detail the mechanical and biological properties of this bi-layered in vitro for skin tissue engineering applications ${ }^{12}$. The effects of the AM/ESF on secretion of angiogenesis 
factors from adipose tissue-derived mesenchymal stem cells (AT-MSCs) are also investigated in vitro.

In this work, we hypothesize that the above fabricated 3D protein-based bilayer artificial skin alone and in combination with adipose tissue-derived mesenchymal stem cells (AT-MSCs) may have a promising impact on angiogenesis and scarless healing of $3^{\text {rd }}$ degree burn wounds in $\mathrm{BALB} / \mathrm{c}$ mice. This idea of utilization of the engineered bi-layer artificial skin is based on our in vitro observations. This throws information about the maintenance of efficacy when the engineered matrix/scaffold is translated from in vitro to in vivo behaviors. This report describes the angiogenesis and scarless healing effects of the AM/ESF bi-layered artificial skin in vivo in mice burn wound model. The results of this experimentation clearly indicate that the engineered blend matrices of this type may be used for quick recovery of deed burn injuries and finally to obtain scarless healing.

\section{MATERIALS AND METHODS}

\subsection{Preparation of decellularized human amniotic membrane/electrospun nanofibrous silk fibroin (AM/ESF)}

The AM/ESF bi-layered 3D artificial skin was prepared and fully characterized in vitro in our recent investigation ${ }^{12}$. Briefly, the AM samples were collected and denuded by a simple and cost-effective method described previously ${ }^{13}$. After confirmation of the full removal of the cells and cells' fragments, AM was covered with ESF with average diameter of $93 \pm 25 \mathrm{~nm}$ by electrospinning. The AM/ESF membrane was embedded in $70 \%$ ethanol for $1 \mathrm{~h}$ to determine the molecular conformation of the $\mathrm{SF}^{14}$. After embedding in ethanol, ESF was tightly coated to AM 
and was not separable. The AM/ESF 3D bi-layered artificial skin was cyto-biocompatible with favorable biomechanical property and angiogenic effects when seeded with AT-MSCs in vitro ${ }^{12}$.

\subsection{In vivo study}

\subsubsection{Animals}

BALB/c male mouse (weighing 16-19 g) were purchased from Pasteur Institute of Iran and then acclimatized in an animal facility under standard conditions for one week prior to use. The surgery was performed according to the 8th edition of the "Guide for the Care and Use of Laboratory Animals" ${ }^{15}$.

\subsubsection{Experimental thermal injury}

The animals were subjected to $3^{\text {rd }}$ degree round skin burns $(1 \mathrm{~cm}$ in diameter $)$ by brass probe as described previously ${ }^{16}$ with minor modification. The animals were anesthetized with an IP injection of ketamine hydrochloride $\left(100 \mathrm{mg} \cdot \mathrm{kg}^{-1}\right)$ and xylazine $\left(10 \mathrm{mg} . \mathrm{kg}^{-1}\right)$ (both from Sigma, USA) and placed in prone position on a surgical frame. The dorsal surface of mice was shaved and sterilized using $70 \%$ ethanol. The heated metal sheet $\left(100{ }^{\circ} \mathrm{C}\right)$ was placed on mice's dorsum for $10 \mathrm{~s}$ with no external pressure to induce consistent 3 rd degree burn injuries. The $3^{\text {rd }}$ degree burn wound was confirmed by histological analyses (Fig. 1c). Two identical burn wounds were produced (left and right sites of the dorsum) for each mouse. 


\subsubsection{Grafting procedure}

The animals were randomly divided into following five experimental groups:

G1 - controls with $3^{\text {rd }}$ degree burn wound and no implantation (No treated wound, NTW), (negative control $(n=15)) ; \mathrm{G} 2-3^{\text {rd }}$ degree burn wound implanted with AM alone $(n=15)$; G3 $-3^{\text {rd }}$ degree burn wound implanted with AT-MSCs and AM (AM/AT-MSCs) construct ( $n=15)$; G4 $3^{\text {rd }}$ degree burn wound implanted with AM/ESF alone $(n=15)$, and G5 - $3^{\text {rd }}$ degree burn wound implanted with AT-MSCs and AM/ESF (AM/ESF/AT-MSCs) construct ( $n=15)$. For experimental groups G3 and G5, $1 \times 10^{4}$ AT-MSCs were cultured on the membranes for 72 hours prior to implantation. The AT-MSCs were isolated from inguinal fat pads of BALB/c mice and fully characterized by flow cytometry (for CD34, CD44, CD45, CD73, CD90 and CD105) and differentiation capacity toward osteogenic and adipogenic lineages in in vitro by a protocol described in details in in vitro part of this study published elsewhere ${ }^{12}$. The cells harvested from second passage were used in this study. For grafting, the post-burning necrotic tissue was cleaned. After debridement, the membranes were placed on the wounds of G2-G5 experimental groups. In G1 group, the wounds were left with no treatment method. The membranes were well adhered to wound bed during 3-4 min post grafting with no suturing. No membrane detachment was observed during the 28 days period. Each wounds only received one membrane after debridement with no graft changing during the interval time points. In it noted that two wounds of each mouse were treated by the same method to avoid systemically effects of one wound to another near wound ${ }^{17}$. For example in G5, both wounds were implanted with AM/ESF-ATMSCs construct. The wound healings were studied by macroscopic observations, histological examinations and RT-PCR. The burn wound model and grafting are illustrated in Figure 1. 


\subsubsection{Macroscopic observations}

Figure 1. Summarized schematic of the current study. (a) Creation of the $3^{\text {rd }}$ degree burn wounds (step 1 to 6), (b) representation of the $3^{\text {rd }}$ degree burn, (c) H\&E sample depicting $3^{\text {rd }}$ degree burn wound (d) generation of the burn wound including re-epithelialization and wound healing cells recruitment. (Yellow arrows indicate the direction of re-epithelialization). AT-MSCs: Adiposederived stem cells; BC: Blood clot; ECs: Epithelial cells; AM: Human amniotic membrane; AM/ESF: Amniotic membrane/Electrospun silk fibroin; HF: Hair follicle; KCs: Keratinocyte cells; MQ: Macrophage; MSCs: Mesenchymal stem cells; ESF: Nanofibrous silk fibroin; PMN: Polymorphonuclear leukocytes.

The burn wounds were photographed at days $0,4,7,14$ and 28 post-implantation and the wound sizes were recorded ${ }^{18}$. The wound closure for each time interval was determined by the following formula (Equation 1): 
Wound Closure $\%=\left[\frac{\text { Wound diameter on day0-Wound diameter on dayn }}{\text { Wound diameter on day } 0}\right] \times 100 \quad$ (Equation 1)

\subsubsection{Histological examinations}

The animals were sacrificed on days 7, 14 and 28 days post-implantation. Tissues were collected for histological evaluations. For each time point, five mice were sacrificed from each group. The burn wound tissue including the wound bed and healthy skin surrounding the wound were collected and fixed with $10 \%$ neutral buffered formalin at room temperature for $48 \mathrm{~h}$. The samples were dehydrated by ethanol, embedded in paraffin blocks and sectioned at $4 \mu \mathrm{m}$ thickness slices ${ }^{13}$. The sections were stained with Hematoxilin \& Eosin (H\&E), Masson's trichrome and immunohistochemistry. Five sections were taken from each paraffin block. For each subsequent measurement, 10 non-overlapping microscopic field-of-views were chosen including 5 fields at the edges and 5 fields at the center of the wound. The H\&E stained sections were investigated for wound healing, re-epithelialization and formation of new hair follicles, scar elevation index (SEI) and epidermal thickness index (ETI). Masson's trichrome stained sections were used to determine the collagen accumulation. Immunohistochemistry was performed to evaluate angiogenesis, wound healing and scar formation. All counts and scoring were performed independently by three blind observers.

\subsubsection{Wound healing scoring}

The wound healing response in $H \& E$ stained samples was scored according to a method published elsewhere ${ }^{19,20}$. The scoring was as follows: "0" represented none to minimal cell 
accumulation and granulation tissue; "1" represented thin and immature granulation dominated by inflammatory cells but with few fibroblasts; "2" depicted moderately thick granulation tissue, ranging from being dominated by inflammatory cells to more fibroblasts and collagen deposition, extensive neovascularization; "3" constituted thick, vascular granulation tissue dominated by fibroblasts and extensive collagen deposition; and "4" amounted to fully healed wound.

\subsubsection{Hair follicles formation}

The average number of the hair follicles (HF) formed in the burned areas was determined in H\&E-stained sections ${ }^{16}$. The average number of hair follicles within ten fields of H\&E stained sections (five fields from center and five fields from margins of wound) was counted for each experimental group.

\subsubsection{Scar elevation index (SEI)}

The H\&E stained samples were viewed at $\times 100$ magnification and the SEI was determined $(n=5)$ for histomorphometric analysis as described elsewhere ${ }^{21,22}$. The SEI was defined by the following formula (Equation 2):

$S E I=\left[\frac{T W D}{N D}\right]$

(Equation 2) 
Where TWD and ND indicate the height of total dermis wound and height of normal underlying dermis (defined based on the height of the adjacent normal dermis), respectively. The SEI of 1 and $>1$ represent scarless wound healing and scar formation, respectively.

\subsubsection{Epidermal thickness index (ETI)}

The H\&E stained sections were observed under light microscope (Olympus BX51, Olympus, Tokyo, Japan) at $\times 400$ magnification and the average thickness of the epidermis across the five fields of scar tissue was measured to calculate the ETI defined by the epidermal hypertrophy degree. The ETI was defined based on a formula (Equation 3) described by RahmaniNeishaboor et al. ${ }^{22}$ :

$E T I=\left[\frac{\text { height of epidermis in scar tissue }}{\text { height of epidermis in normal uninjured skin }}\right]$

(Equation 3)

The epidermal thickness from five fields of uninjured skin was also measured from both sides of the scar tissue ${ }^{22}$. The ETI of 1 and $>1$ indicate fully healing of wound without scar formation and newly formed hypertrophied epidermis, respectively.

\subsubsection{Masson's Trichrome}

The sections collected from days 7, 14 and 28 were stained with Masson's trichrome and viewed under light microscope (Olympus BH-2, Japan) for the assessment of collagen content and maturation within the dermis. The collagen deposition was scored from grade 0 (lowest collagen density) to grade 4 (highest collagenesis). 


\subsubsection{Immunohistochemistry}

The expressions and densities of collagen types I, III and IV (Col I, III and IV) at day 28, CD31 (PECAM-1) at days 14 and 28, and VEGFa1 and VEGFR2 at days 7, 14 and 28 were studied by immunohistochemistry ${ }^{21,23,24}$. The paraffin-embedded sections were re-hydrated through a graded series of ethanol and the endogenous peroxidase was inactivated by $3 \% \mathrm{H}_{2} \mathrm{O}_{2}$. The antigen retrieval was performed by heating method using an autoclave. After blocking the nonspecific binding sites with $5 \%$ bovine serum albumin (BSA) for $1 \mathrm{~h}$, the samples were incubated with primary antibodies against Col I, III, IV, CD31, VEGFa1 and VEGFR2 (all purchased from Abcam, Cambridge, MA, USA) at $4{ }^{\circ} \mathrm{C}$. The sections were then treated with antirabbit/anti-mouse Envision (Dako, Denmark) as a secondary antibody for $30 \mathrm{~min}$. The samples were visualized by incubation with a 3,3'-diaminobenzidine (DAB) solution as substrate and viewed under light microscope (Olympus BX51, Olympus, Tokyo, Japan) at $\times 100$ magnification ${ }^{25}$. Weidner's method was used for determination of micro-vessel density (MVD) using CD31 expression ${ }^{21}$. For this, the most vascularized areas within the CD31 immuno-stained samples of each experimental group were selected at $\times 100$ magnification and the microvessels (vessels with distinct lumens) were counted within each selected area at $\times 200$ magnification. The MVD values were reported as the average count of microvessels in three high vascularized areas. All the scoring and counts were performed by three independent observers blinded to the experimental setup.

\subsubsection{Real-time PCR}


The tissue samples $(50 \mathrm{mg})$ were harvested at days 7, 14 and 28 post-implantation and the total RNA was extracted using an RNeasy Mini Kit (Cinagen, Tehran, Iran) following the Supplier's instructions. The full thickness skin tissue away from the implantation site served as control and coded as "normal skin" (NS). In brief, the samples were trimmed of excessive surrounding tissue, cut into small pieces and lysed with lysing buffer. The cDNA was synthesized using random hexamer primers by a High-Capacity cDNA Archive kit (Applied Biosystems, Foster City, CA, USA) following the Supplier's instructions. The relative transcript levels of Collagen type I, Collagen type III, Collagen type IV, TNF $\alpha$, TGF- $\beta 1$, MIP-2, IL-1b, bFGF, VEGF- $\alpha 1$, VEGF-R2, MMP-1 and MMP-2 were determined by rotor-gene 6000 (Corbett Life Science, Sydney, Australia) instrument using PCR Master Mix (TaKaRa, Dalian, China). The conditions of the reactions were as follows: $95^{\circ} \mathrm{C}$ for $10 \mathrm{~min}$ followed by 40 cycles of $95{ }^{\circ} \mathrm{C}$ for $15 \mathrm{~s}, 60{ }^{\circ} \mathrm{C}$ for $30 \mathrm{~s}, 72{ }^{\circ} \mathrm{C}$ for $30 \mathrm{~s}$. The $\mathrm{Ct}$ value for each sample was defined. Relative transcript level for each target gene was normalized against beta actin (b-actin) as housekeeping gene. The relative expression level fold was defined using $2^{-\Delta \Delta C t}$. Tissue samples harvested from areas with no wounds served as negative controls. All primers used in this study and the product lengths are listed in SI Table 2.

\section{RESULTS AND DISCUSSION}

\subsection{In vivo observations}

\subsubsection{Macroscopic observation and wound closure}

The photographs of the wounds at $0,4,7,14$ and 28 days post-wounding are shown in Figure

2a. As anticipated, there was a significant difference in wound contraction between the 
implanted experimental groups (G2-5) and NTW (G1). Almost all the wound areas were reepithelialized in groups G3-G5 at day 14. The recorded wound sizes and the percentage of wound closure were determined and compared between all experimental groups (Figure $\mathbf{2 b}$ ). The early wound closure is of the most crucial parameters in treatment of wounds, especially the extensive ones. Silk protein was found to enhance the wound closure and improve healing ${ }^{26}$. As shown in Figure 2, there was a significant difference between the wound closure levels in the animals implanted with AM/ESF/AT-MSCs when compared to those with the animals implanted with AM/AT-MSCs on days 7,14 and 28 post-surgery $(\$ p \leq 0.05)$. This suggests that the seeded mesenchymal stem cells had an important effect on proliferation, migration and differentiation. Moreover, the wound sizes in the animals treated with AM/ESF were significantly smaller when compared to those treated with AM on day 14 and $28(\uparrow \mathrm{p} \leq 0.05)$. The wound sizes showed statistically significant decrease in all the implanted animal groups in comparison with the NTW group (G1) on days 7, 14 and 28. The presence of ESF had a profound effect in wound healing acceleration when compared to the AM only. The positive effects of the ESF in wound acceleration were reported by others ${ }^{27-29}$. 


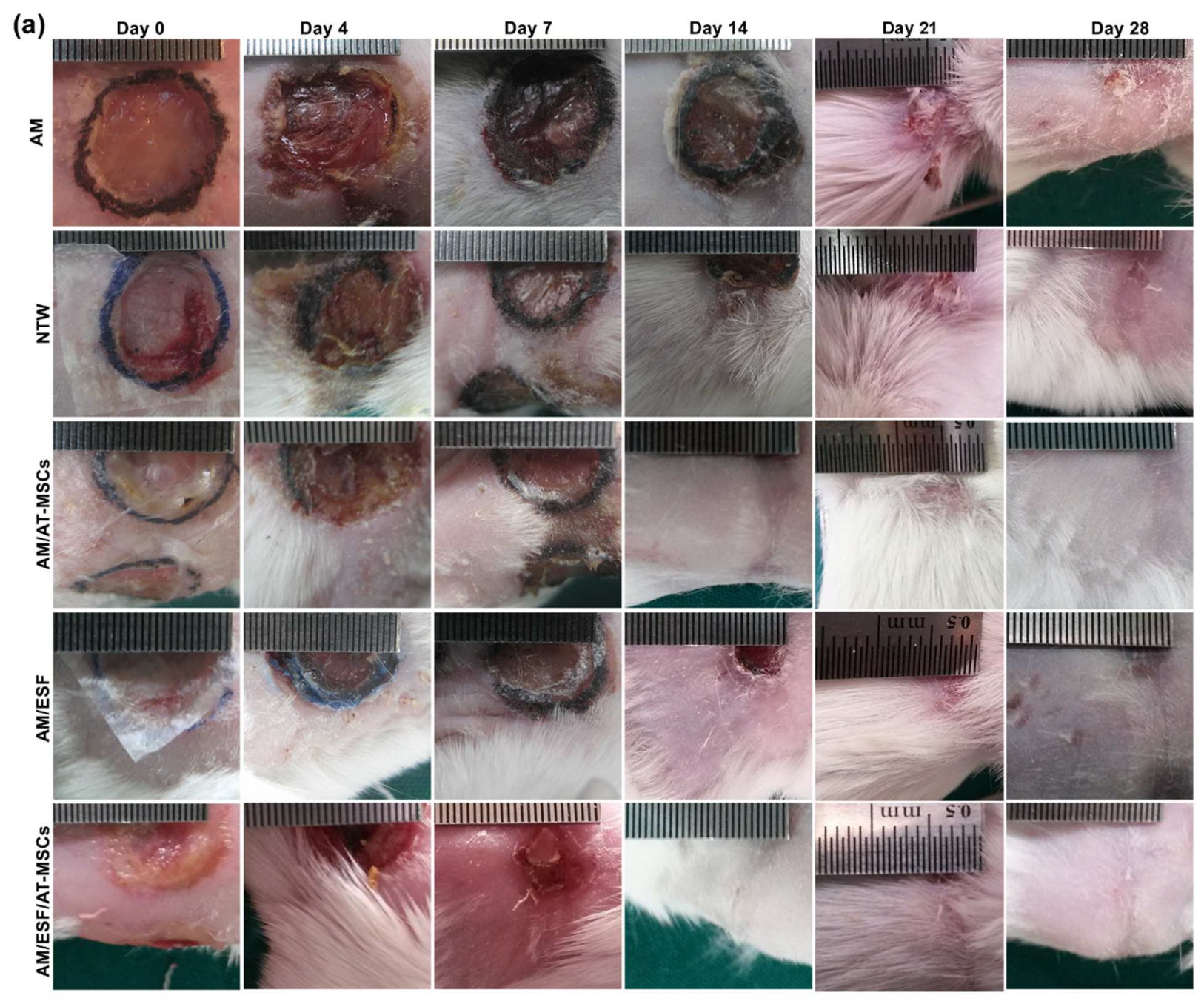

(b)

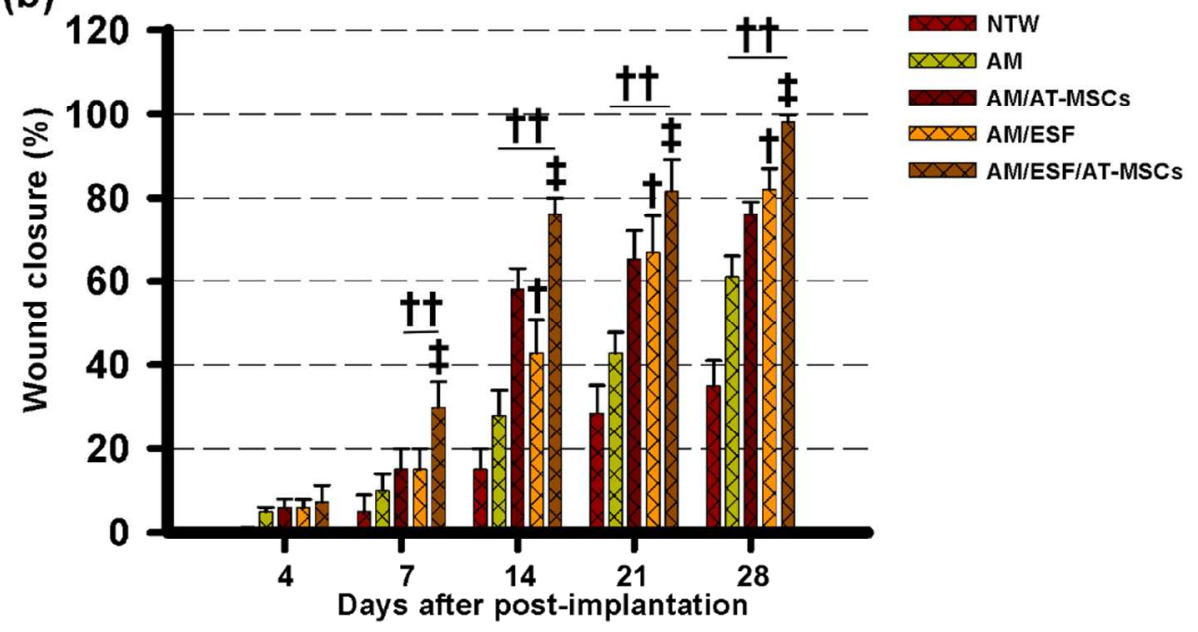


Figure 2. Macroscopic observations and wound closure. (a) The photographs of wounds in BALB/c mice in which the wounds received implantation of AM (G2), AM/AT-MSCs (G3), AM/ESF (G4) and AM/ESF/AT-MSCs (G5). The animal with burn wound and no implantation served as control group (NTW, G1). Representative photographs of the wounds were taken at 0 , 4, 7, 14 and 28 days post-wounding. (b) Percentage of wound closure in all experimental groups at $0,4,7,14$ and 28 days post-wounding. $+\dagger$ statistically significant difference with the NTW group $(\mathrm{G} 1)$; $\uparrow$ statistically significant difference with the animals received AM (G2); + statistically significant difference with the animals received the same implant without AT-MSCs (G2). The data are analyzed using one-way ANOVA (post hoc: LSD), as $\mathrm{p} \leq 0.05$ is considered as level of significance. Each bar represents mean $\pm \mathrm{SD}$.

\subsubsection{Histological examinations}

\subsubsection{1. $H \& E$}

The photographs of the H\&E sections are shown in Figure 3a. At day 7 post-wounding, the reepithelialization began from the margins of the wound. In addition, infiltration of the PMN and neutrophils was clearly observed in all experimental groups. The amount of acute inflammatory cells in the wound area of the NTW group was very low when compared to other experimental groups. The stretch of the membrane over the underlying muscle tissue is clearly visible. The granulation as well as deposition of extra-cellular matrix elements in the animals receiving implants was obviously higher than that in NTW group (G1). No hair follicles were observed within the burned area (Figure 3c). The histological score of the AM/ESF/AT-MSCs treated animals was significantly higher than other experimental groups at day $7(\mathrm{p}<0.01)$. At this point 
of time, the healing in all the implanted animals (G2-4) were better than NTW $(\mathrm{p}<0.05)$ (Figure 3b).

On day 14, all the surfaces of the wound were occupied by the epidermis layer. The newly formed granulation tissue was continued to be thicker and the cells were well penetrated into the wound site. At that time, hair follicle formation is clearly observed in margins of the wounds. Granulation and cellularity in G4 and G5 was higher than in the G2 and G3, respectively. In experimental groups G3-G5, the healing was followed by the maturation of the granulation tissue (Figure 3a). After 14 days post-operation, the difference between the healing scores of the experimental groups (G3-5) was significantly higher than those in NTW animals $(p<0.01)$ (Figure 3b).

At 28 days post-implantation, the wounds in G1 and G2 remained unhealed, while almost all the wound areas were healed in other experimental groups (Figure 3a). The structure and appearance of the skin tissue in the middle of the defect was closely similar to the normal skin (NS) in G5. Hair follicles were appeared in the middle of the AM/ESF/AT-MSCs-treated wounds. With maturation of granulation tissues, the cellularity in the defect sites of the animals receiving the AM/AT-MSCs, AM/ESF and AM/ESF/AT-MSCs was profoundly decreased (Figure 3a). The histological observations of the H\&E stained-sections revealed the positive effects of the ESF-covered AM and also the presence of AT-MSCs in promotion of skin tissue healing. In a separate study, AT-MSCs were seeded on silk fibroin/chitosan (SF/CS) and then implanted in Murine Soft Tissue Injury Model $^{30}$. A significant acceleration in wound healing of AT-MSCs-silk fibroin/chitosan receiving wounds was observed when compared to untreated animals or treated with SF/chitosan. The effects of SF on skin wound healing was further showed by Shefa et al. ${ }^{31}$. They fabricated an oxidized cellulose nanofiber-SF porous scaffold and 
showed that this scaffold had superior in vitro and in vivo characteristics for critical sized rat skin excisional wound healing compared to oxidized cellulose nanofiber alone.

Our results show that both SF and AT-MSCs remarkably accelerate burn wound healing through early re-epithelialization and ECM formation. At day 28 , the wound healing scores in the AM/ESF receiving animals was significantly higher than AM alone. Moreover, all the treated animals had a significantly accelerated wound healing score in comparison to the NTW group $(\mathrm{p}<0.05)$ (Figure 3a).

The average number of the newly formed hair follicles in the wounds implanted with AM/ESF and AM/ESF/AT-MSCs was significantly higher than those in AM and AM/AT-MSCs at days 14 and $28(\mathrm{p}<0.05)$. This indicates the favorable effects of both ESF and AT-MSCs on formation of hair follicle. Furthermore, there was no significant difference between mean numbers of newly formed hair follicle in AM/ESF/AT-MSCs implanted wound (G5) and NTW (G1). It is reported that the formation of new hair follicles was not required for a complete healing of skin wounds. This may significantly accelerate the healing process ${ }^{22,32}$.

The histological data of the current study were in agreement with other investigations, which used mesenchymal stem cells and silk fibroin in skin tissue regeneration ${ }^{27,33,34}$. For instance, bone marrow-derived mesenchymal stem cells enhanced skin wound healing though differentiation and angiogenesis ${ }^{27}$. According to other reports, silk fibroin protein secretes specific growth factors, which aid wound healing. SF enhance wound healing through differentiation and proliferation of mesenchymal stem cells and therefore facilitate wound healing ${ }^{30,35,36}$. 
(a)
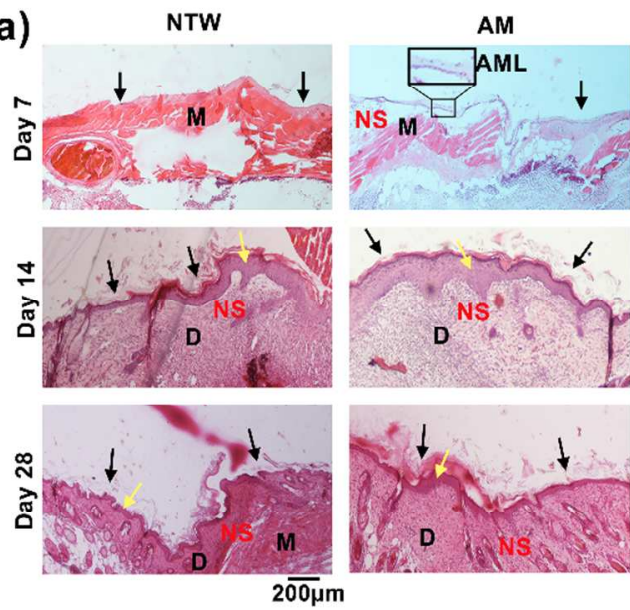

(b)

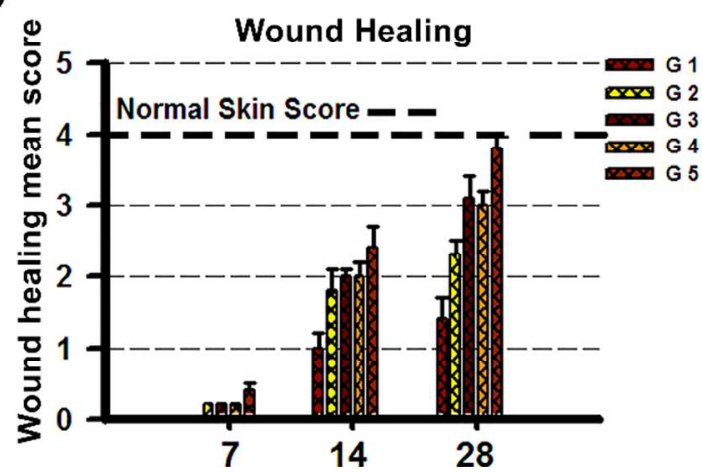

Days after post-implantation

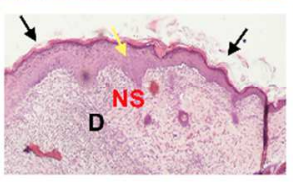

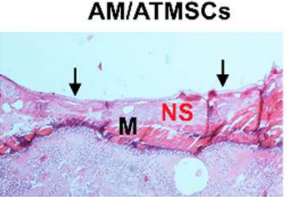
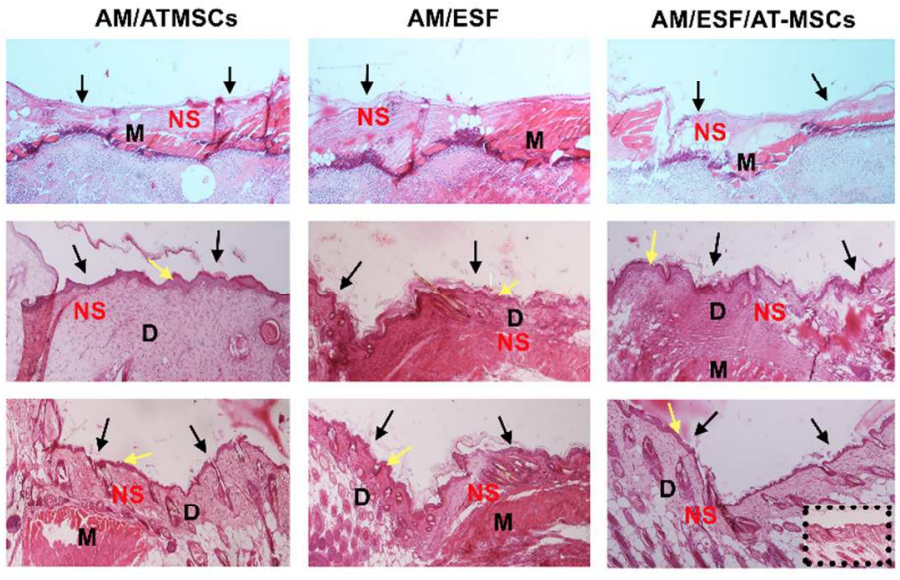

(c)

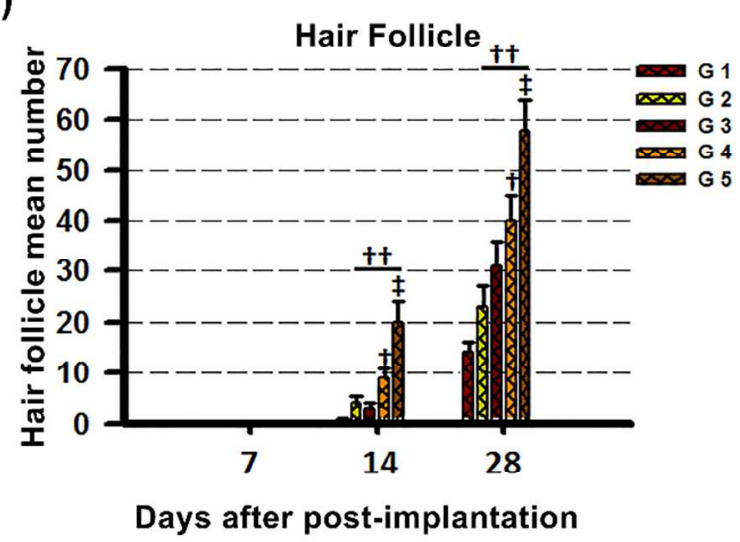

Figure 3. Histological observations. (a) Hematoxylin and eosin stained samples at days 7, 14 and 28 post-surgery. (b) Wound healing scoring and (c) mean hair follicle formation. D: Dermis; M: Muscle; AML: Amniotic membrane layer; NS: Newly formed skin. Insert: Unwounded skin. Black and yellow arrows indicate outer surface of skin and epidermis layer, respectively. $\$$ indicates significant difference with $\mathrm{G} 3$; $\uparrow$ indicates significant difference with $\mathrm{G} 2$; $\dagger \dagger$ indicates significant difference with G1 (NTW) $(\mathrm{p}<0.05)$.

\subsubsection{Collagen synthesis and density}


Collagen deposition within the burned tissues at days 7, 14 and 28 post-implantation was determined by Masson's trichrome staining. Collagen and cell nuclei were stained in blue/green and dark blue, respectively (Figure 4a). On day 7, the collagen deposition in the wounds treated with AM/ESF/AT-MSCs was much higher than other experimental groups. After 14 days, the NTW control and AM implanted animals showed highest collagen deposition compared with other groups. Collagen density in the NTW control and AM remained high at day 28 postsurgery, while the wounds treated with AM/AT-MSCs, AM/ESF and AM/ESF/AT-MSCs were similar to that in NTW (Figures 4a and insert). All the Masson's trichrome stained samples indicated that both ESF and AT-MSCs significantly accelerated wound healing. As shown in Figure 4a the wounds treated with AM/ESF/AT-MSCs healed almost completely on day 28 . To confirm the results obtained from Masson's trichrome staining, the relative expressions of collagen types I, III and IV at days 7, 14 and 28 post-operation were determined by RT-PCR. The results were compared among all the experimental groups (Figure $4 \mathbf{b}$ ). The results confirmed that all the treated groups, especially AM/ESF and AM/ESF/AT-MSCs, exhibited change in collagen type from COL III to COL I by day 14 . This suggests that wound healing was accelerated in these experimental groups. In a study by Shan et al. ${ }^{20}$ the silk fibroin/gelatin nanofibrous skin dressing promoted the burn wound healing by regulating newly formed collagens and improving collagen organization. Our results are consistent with other relevant studies $^{20,33,37-39}$. 

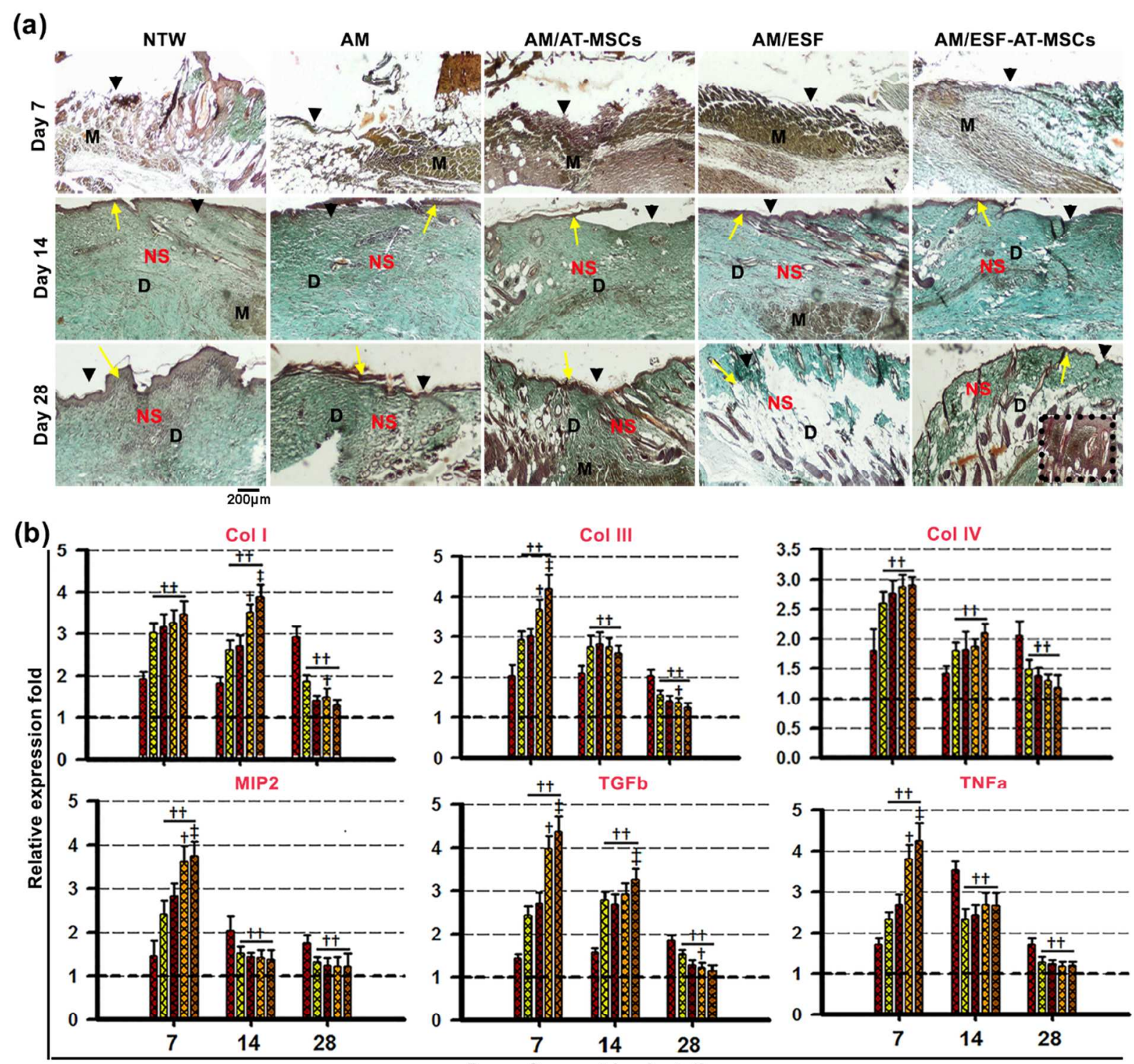

Days after post-implantation

Figure 4. Determination of collagenesis. (a) Masson's trichrome staining of the different samples at days 7, 14 and 28 post-operation. (b) Collagen deposition scoring, relative expression of COL I, III and IV at 7, 14 and 28 days after post-operation. The expression results was normalized to that in normal skin. Relative expression of MIP2, TNF $\alpha$ and TGF $\beta 1$ in defect tissue of animals at 7, 14 and 28 days after post-implantation. The expression results are normalized to that of normal skin. D: Dermis; M: Muscle; NS: Newly formed skin. Insert: Unwounded skin stained with Masson's trichrome. Black triangle and yellow arrow indicate 
outer surface of skin and Epidermis layer, respectively. $\$$ indicates significant difference with G3; $\dagger$ indicates significant difference with G2; $\dagger$ i indicates significant difference with G1 $(\mathrm{NTW})(\mathrm{p}<0.05)$.

\subsubsection{Localized inflammatory response}

The relative expressions of MIP2, TNF $\alpha 1$ and TGF $\beta 1$ at 7, 14 and 28 days of post-implantation were determined by RT-PCR to verify whether the implanted membrane stimulates any inflammatory reactions. The results are shown in Figure $\mathbf{4 b}$. TNF- $\alpha$ is secreted by various cell types, especially activated macrophages. It is activated in acute phase inflammation through regulation of inflammatory cells. TGF- $\beta 1$ is secreted by many cell types, especially leucocytes ${ }^{8}$, 40, 41. TGF- $\beta 1$ promotes wound healing through regulation of cell growth, migration, differentiation and apoptosis ${ }^{8,42}$. MIP2 (called also chemokine (C-X-C motif) ligand 2) is secreted by monocytes and macrophages. This cytokine is a chemoattractant for PMN and causes localized inflammatory reactions ${ }^{8,43}$. According to our results, all the analyzed cytokines were significantly upregulated in all animals with implanted wounds compared to NTW control at day $7(\mathrm{p} \leq 0.05)$. At the same time, an increased expression level of these cytokines in the AM/ESF and $\mathrm{AM} / \mathrm{ESF} / \mathrm{AT}-\mathrm{MSC}$ s treated wounds was observed in comparison with other experimental groups. At day 14, TGF- $\beta 1$ remained upregulated in all treated wounds, while MIP2, TNF $\alpha 1$ were significantly down-regulated when compared to NTW group $(p \leq 0.05)$. The MIP2, TNF $\alpha 1$ and TGF $\beta 1$ gene expression levels were returned to normal level in all treated wounds by day 28. These results apparently revealed that the inflammatory response was not completely eliminated in NTW group event by day 28. A possible explanation of this is that the ESF accelerate wound 
healing through inciting the acute phase immune response (PMN) and recruitment of macrophages into defect site during the first days of implantation. We also observed an immunomodulatory activity of the AT-MSCs at day 14 of post-operation.

\subsubsection{Vascularization}

Neovascularization is an essential step in obtaining successful healing of a wounded skin. Delayed neovascularization often leads to delayed wound healing and formation of a fibrotic tissue causing a significant drawback when it comes to the available skin substitutes today ${ }^{44,45}$. Earlier, we found that AM/ESF up-regulate the expression of angiogenic factors such as VEGFal and bFGF in AT-MSCs in vitro ${ }^{12}$. To evaluate the effects of ESF and AT-MSCs on promoting neovascularization in vivo, the expression and localization of VEGFa1, VEGFR2 and CD31 were determined by immunohistochemistry and scoring analyses (Figures 5 and 9). VEGFa is one of the most important angiogenesis factors. It promotes angiogenesis by acting on endothelial cells 8, 46, 47 via VEGFR2 that is highly expressed on these cells ${ }^{48,49}$. The Immunohistochemistry results of the VEGFa and VEGFR2 are depicted in Figures 5a, 5b and 6. At post-operation day 7, the expressions of VEGFa1 and VEGFR2 in all the treated experimental wounds were higher than that in NTW group. At this time, the expression of these factors in the wounds treated with $\mathrm{AM} / \mathrm{ESF}$ and AM/ESF/AT-MSCs was much higher than in other experimental groups. At day 28 post-operation, the expressions of VEGFa and VEGFR2 were profoundly down-regulated in all treated groups compared to NTW group. Li et al ${ }^{50}$ fabricated a strontium loaded SF/sodium alginate film with high cytocompatibility and proangiogenesis action for skin wound healing applications. They showed that loading strontium to SF blend 
increased secretion of VEGF and bFGF form L929 fibroblasts in vitro and may successfully promote neo-vascularization in wound bed.
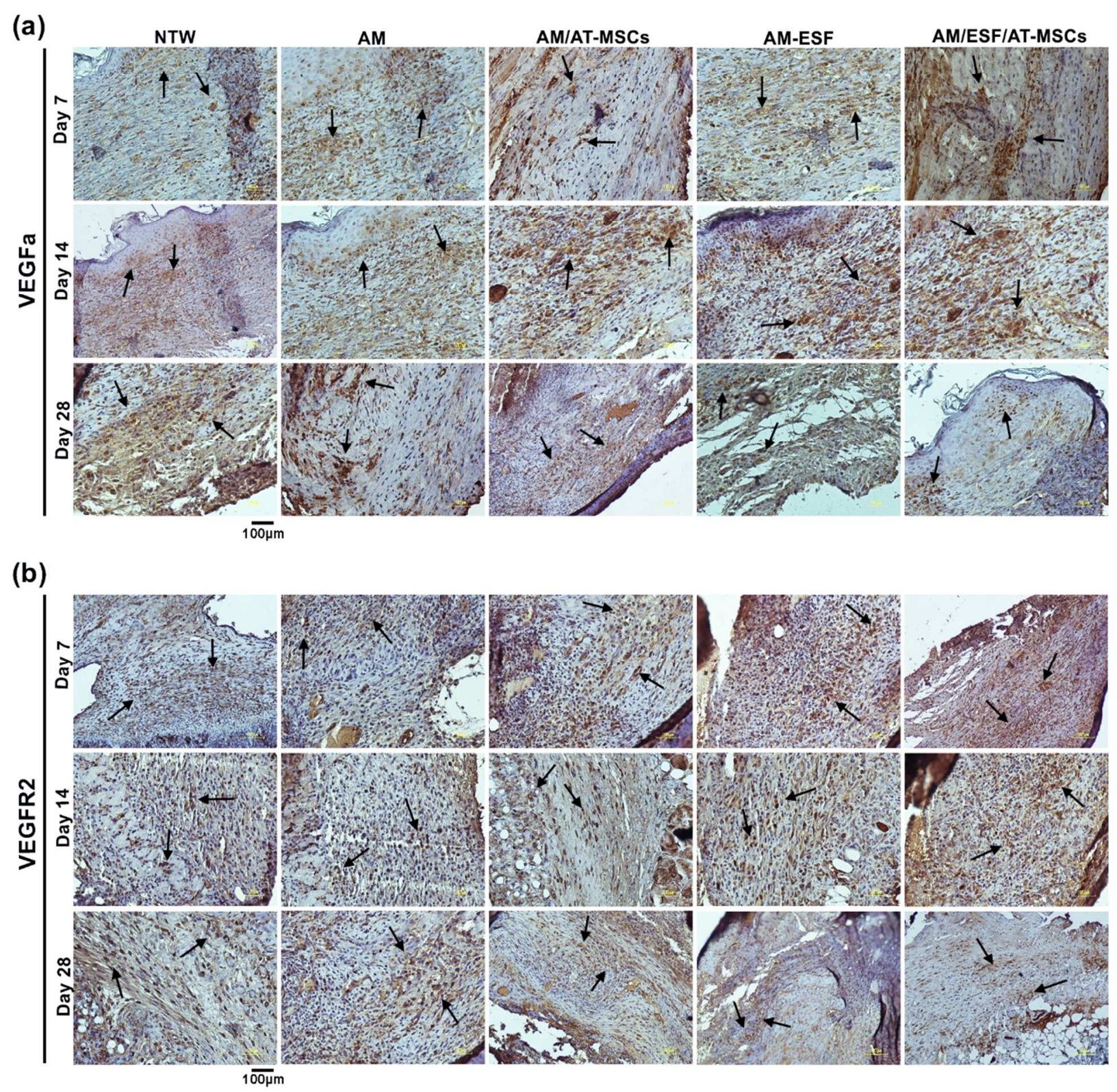
Figure 5. Neovascularization assay. a) Immunohistochemistry staining of VEGFa and (b) VEGFR2 at 7, 14 and 28 days of post-operation. The black arrows indicate VEGFa and VEGFR2. NTW (wound with no implantation).

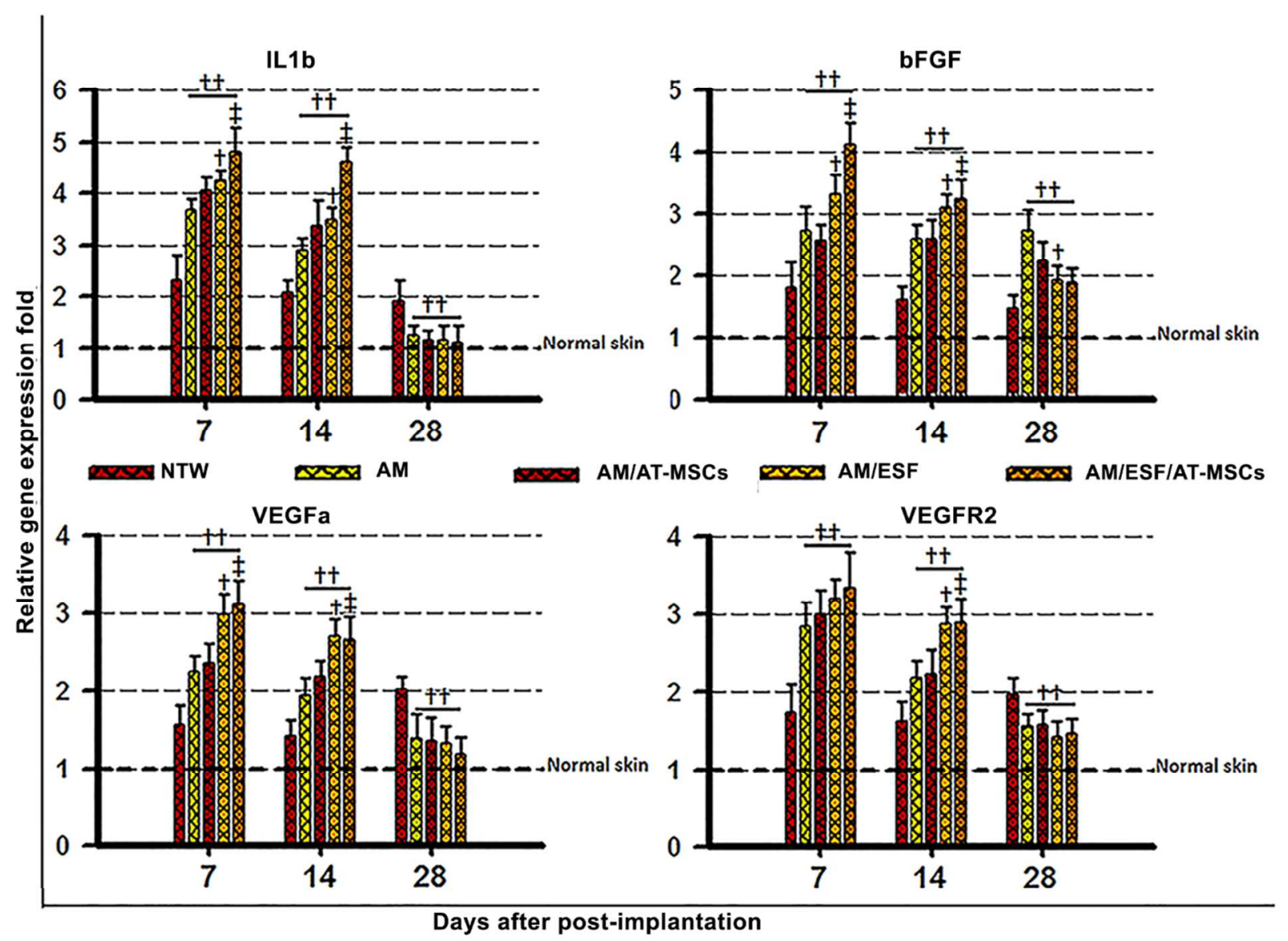

Figure 6. RT-PCR results of relative expressions of IL1b, bFGF, VEGFa and VEGFR2 at 7, 14 and 28 days of post-operation. $\$$ indicates significant difference with G3; $\uparrow$ indicates significant difference with G2; †† indicates significant difference with G1 (NTW). NTW (wound with no implantation); NS (normal skin). 
The expression of CD31, a specific marker of endothelial cells of early blood vessels, was also evaluated by immunohistochemistry to determine the microvascular density (MVD) within wound sites ${ }^{21,51}$. The values of immunohistochemistry results of CD31 and MVD are shown in Figures $7 \mathbf{a}$ and $7 \mathbf{b}$, respectively. At day 7, no blood vessels were observed in any of the wounds. At 14 days of post-operation, the MVD values in all the treated wounds were much higher than in NTW group. The wounds treated with AM/AT-MSCs, AM/ESF and AM/ESF/AT-MSCs showed the highest MVD value compared to other experimental groups. This suggests that the neovascularization greatly was accelerated during this time interval. At post-implantation day 28 , the wounds treated with AM/AT-MSCs, AM/ESF and AM/ESF/AT-MSCs showed the highest MVD values compared to other groups. 


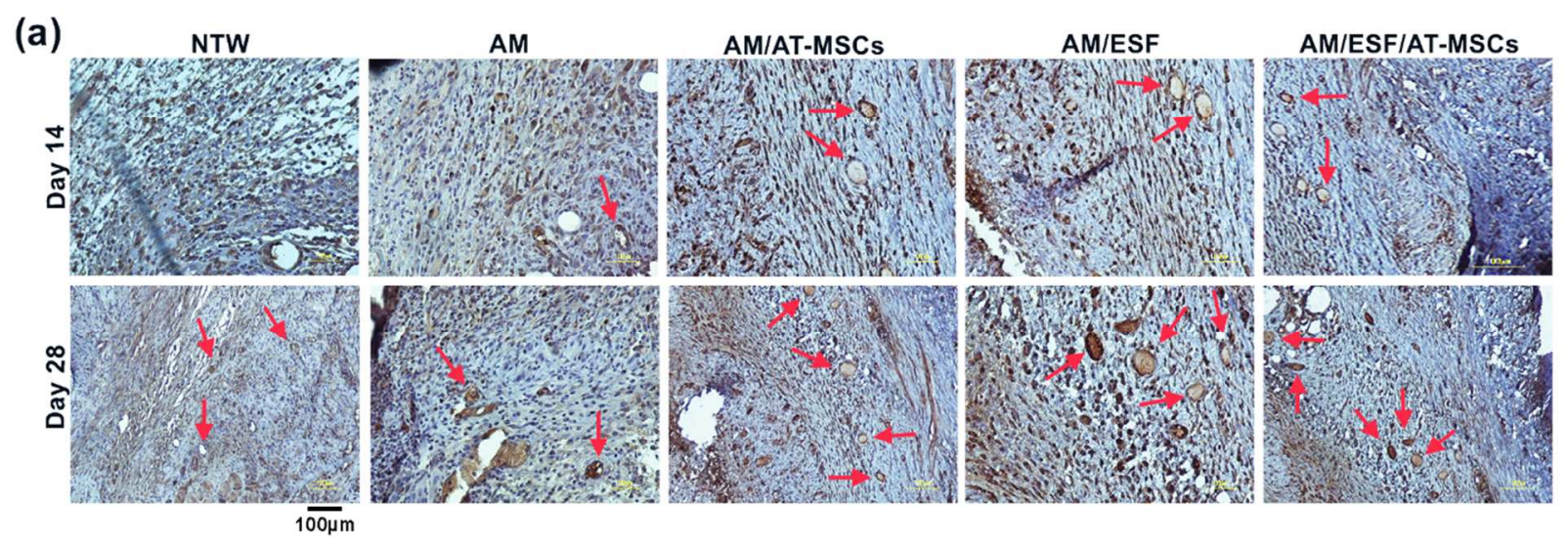

(b)

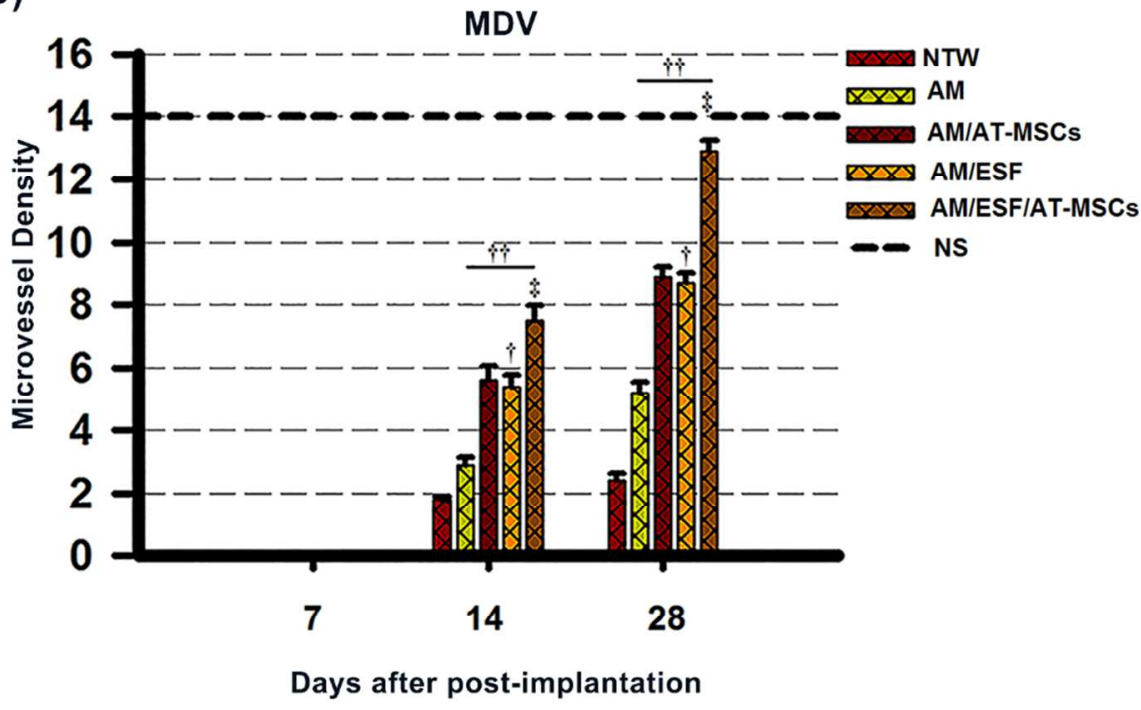

Figure 7. Determination of microvessel density (MVD). (a) Immunohistochemistry staining of CD31 (PECAM-1) at 14 and 28 days of post-operation. (b) MDV of each experimental group on post-operation 7, 14 and 28 days. The yellow arrow indicates micvrovessel. NTW (wound with no implantation); NS (normal skin). + indicates significant difference with $\mathrm{G} 3$; $\uparrow$ indicates significant difference with G2; †† indicates significant difference with G1 (NTW).

We used RT-PCR to study the neovascularization rate in defect site by determining the relative expressions of IL-1 $\beta$, FGF, VEGFa and VEGFR2, the most important factors involved in 
angiogenesis ${ }^{52-54}$. VEGFa is primarily secreted by mesenchymal stem cells ${ }^{55}$. IL-1 $\beta$ is mostly secreted by activated macrophages ${ }^{54,56}$. The relative expressions of the mentioned angiogenic factors were compared with those in normal skin. The results showed that the relative expression of all the examined angiogenic factors were up-regulated in all treated wounds compared to NTW group on days 7 and 14 post-operation. The rate of up-regulation was significantly higher in $\mathrm{AM} / \mathrm{ESF}$ and $\mathrm{AM} / \mathrm{AT}-\mathrm{MSC}$ s treated wounds groups when compared to other groups. At day 28 , the relative expressions of these factors in all treated wounds were significantly downregulated compared to NTW group. The relative expressions of VEGFa and IL-1 $\beta$ returned to that of the levels detected in normal skin were observed.

The SF is an excellent supporter for endothelial cell proliferation, attachment, migration, differentiation and formation of microvessel-like structure ${ }^{57-62}$. Furthermore, the involvement of mesenchymal stem cells in promotion of neovascularization is widely reported ${ }^{27,63,64}$. For instance, Shan et al. ${ }^{20}$ showed that the silk fibroin/gelatin nanofibrous bio-scaffold promoted the burn wound healing process by accelerating angiogenesis. The data obtained from this study indicate that ESF has a great potential in tissue engineering by promoting wound healing process through the increased secretion of angiogenesis factors from AT-MSCs and acceleration of early neovascularization.

\subsubsection{Scar formation}

The samples harvested from day 28 of post-implantation were examined for scar formation. . The samples were measured for collagen deposition, SEI, ETI, gene expression profile of Col I, Co III, Col IV, MIP-2, TGF $\beta 1$, TNF $\alpha 1$, MMP-1 and MMP-2. 


\subsubsection{Re-epithelialization and collagen deposition}

Abnormally excessive collagen deposition in defect site is the most important indicator of scar. The late re-epithelialization and infection increase cellularity within the $3^{\text {rd }}$ degree wounds, retain collagen synthesis, postpone collagen remodeling, and subsequently the accumulation of irregular collagen fibers stimulate scar formation $5,65,66$. The collagen deposition and relative collagen density on day 28 are presented in SI Figures 1a and 1b. The results obtained from Masson's trichrome staining indicated that the collagen density in the wound treated with AM/ESF/AT-MSCs (G5) was reduced. The morphology of the fibers was similar to the normal skin and required more time to complete remodeling. This result is in agreement with earlier report that showed the anti-scarring effects of astragaloside IV-functionalized silk fibroin/gelatin electrospun nanofibrous scaffold ${ }^{20}$. It was also reported that the matrices used as skin dressing material promoted scarless healing of deep partial-thickness burn wound by improving collagen organization. In a study conducted by Acharya et al. ${ }^{67}$, SF was successfully conjugated with lactose in two 2D and 3D scaffold forms to prevent scar formation through suppression of fibroblast differentiation into contractile myofibroblasts. Their results showed that lactoseconjugated SF scaffold has superior cell adhesion property for fibroblasts than myofibroblasts compared to pure SF. Myofibroblasts lost their contractile phenotype when cultured on lactoseconjugated SF compared to SF alone.

Our histological observations showed that re-epithelialization in experimental groups G3, G4 and G5 were $>95 \%,>90 \%$ and 100\%, respectively (SI Figure 1b). All the results revealed positive 
effects of both ESF and mesenchymal stem cells on wound healing, re-epithelialization and prevention of scar that are consistent with earlier studies ${ }^{18,27,68}$.

\subsubsection{Scar elevation index (SEI)}

Scar elevation index results are presented in SI Figure 1b. The wounds showed various rates of scar in all experimental groups. NTW exhibited the highest mean SEI $(2.9 \pm 0.45)$ compared to treated wounds $(\mathrm{p}<0.05)$. SEI for the wounds treated with AM and AM/ESF was $2.1 \pm 0.35$ and $1.6 \pm 0.31$, respectively $(\mathrm{p}<0.05)$. Additionally, the wounds implanted with AM/AT-MSCs and AM/ESF/AT-MSCs had a SEI of $1.5 \pm 0.22$ and $1.1 \pm 0.12$, respectively $(\mathrm{p}<0.05)$. The smaller SEI value represents lower scar formation. Normal skin has a SEI of 1. Significantly reduced SEI values in our experiments indicate a clear reduction of scar in the treated wounds compared to negative controls (NTW). The addition of ESF and AT-MSCs further reduced scar formation by decreasing the thickness of the epidermis. Reduced SEI by other biodegradable scaffolds were reported earlier ${ }^{21,22}$. Cheng et al. ${ }^{21}$ functionalized the ginsenoside-Rg3-loaded fibrous with bFGF growth factor. They observed that the resulted bio-functionalized scaffold reduced SEI in rabbit ear model full thickness wounds. The evidences obtained from SEI results of our study reveal that the silk fibroin nanofibers may reduce scar formation through decrease of dermis proliferation.

\subsubsection{Epidermal thickness index (ETI)}


Epidermal thickness index for all the wounds was measured at 28 days of post-operation and the results are illustrated in SI Figure 1b. The NTW group showed the highest ETI $(4.3 \pm 0.63$ time greater than normal skin) compared to the treated animals' wounds $(p<0.05)$. ETI for the wound treated with AM, AM/AT-MSCs, AM/ESF and AM/ESF/AT-MSCs was $3.2 \pm 0.72,2.1 \pm 0.41$, $1.9 \pm 0.43$ and $1.1 \pm 0.15$, respectively. Our ETI results for a combination of AM and ESF treatment decreased significantly scar formation $(\mathrm{p}<0.05)$. This combinatorial approach of both AM membrane, ESF and mesenchymal stem cells yielded superior results. This greatly inhibits scar formation $(\mathrm{p}<0.05)$. Less epidermal proliferation in the wounds treated with nanofibrous scaffolds and anti-inflammatory factors were reported elsewhere ${ }^{19,21,22}$. For example, RahmaniNeishaboor et al. ${ }^{22}$ impregnated acetyl salicylic acid, as an anti-inflammatory agent, into carboxymethyl cellulose gel and applied topically to rabbit ear model wounds. They observed that topical application of such anti-inflammatory agent profoundly reduced epidermal proliferation and thickness. This resulted in reduced scar formation.

\subsubsection{Gene expression profile}

Scarless healing of wound requires a precise balance between ECM accumulation and degradation. In the late stage of skin wound healing, the majority of deposited collagens is collagen I. In remodeling phase, content, type and orientation of collagens are modified ${ }^{5,69,70 .}$ The depositions of collagen I, III and IV in all the samples at 28 days post-treatment are presented in Figure 8a. The accumulations of all the examined collagen types were highest in the NTW as compared to other experimental groups. From the Immunohistochemistry staining, we observed that the collagens in the scar of the NTW groups arranged randomly. This indicates 
that the NTW was not completely healed and needed more time to be remodeled. The content of the collagen in the animals receiving $\mathrm{AM} / \mathrm{ESF}$ and AM/ESF/AMCSs seemed to be lower than in $\mathrm{AM}$ and AM/AT-MSCs treated wounds. On the other hand, the collagen depositions in all the treated wounds (G2-G5) showed to have a regular orientation. The collagen deposition over the wound in an irregular orientation during the first one week of post-wounding was observed in earlier studies. During remodeling phase, the collagens are degraded and re-arranged in a regular orientation 5, 22, 66, 69 . All the Immunohistochemistry results reveal the positive effects of both ESF and AT-MSCs on decreasing the scar formation.

As mentioned above, overexpression of collagen and subsequent collagen deposition is an important and reliable indicator of scar formation $23,69,71$. The results indicated that the expressions of Col I, III and IV were significantly down-regulated in the implanted animals compared with NTW (Figure 8b). The animals treated with AM and NTW showed a significant up-regulation of Col I gene expression as compared to other experimental groups and NS. The expression of Col IV in the scar site of NTW was higher than those in other groups $(\mathrm{p} \leq 0.05)$. The expressions of $\mathrm{Col}$ I and IV were up-regulated in experimental groups G2 to G5. The difference was not significant when compared to NS. On the other hand, the expression of Col III in animals treated with AM and AM/ESF as well as NTW remained significantly up-regulated as compared to NS $(\mathrm{p} \leq 0.05)$. All the results revealed the effects of the AM/ESG grafting on downregulation of collagen gene expression. This leads to a conclusion that the scar formation was remarkably decreased. The presence of the AT-MSCs significantly decreased the scar formation through down-regulation of the collagen gene expression. It is reported that the AT-MSCs have anti-inflammatory properties through the secretion of several anti-inflammation factors ${ }^{72}$, ${ }^{73}$.Spiekman et al. ${ }^{42}$ demonstrated that adipose tissue-derived stromal cells prevented scar 
through inhibition of TGF- $\beta 1$-induced adverse differentiation of human dermal fibroblast in a paracrine fashion.

To further understand how the AT-MSCs, ESF and AM reduce the scar formation, the expressions of TNF- $\alpha$ and TGF- $\beta 1$ were compared between the experimental groups at 28 days of post-implantation. The expression of MIP-2, TNF- $\alpha$ and TGF- $\beta 1$ in the animals implanted with AM and NTW remained significantly up-regulated when compared to untreated control $(\mathrm{p} \leq 0.05)$. In addition, expression of TNF- $\alpha$ in the AM/ESF group was significantly lower than in wound treated with AM (Figure 8b).

The results of this study indicated that both AT-MSCs and ESF might assist in lowering of the scar formation through minimizing inflammatory response and subsequently decreased collagenesis. This was in agreement with a study that showed the effects of ginsenoside-Rg3loaded fibrous PLGA functionalized with bFGF growth factor on prevention of scar though a decrease of collagen deposition ${ }^{21}$.

Another important indicator of the scar formation is the presence of matrix metalloprotease (MMP) activity. MMPs are diverse proteolytic enzymes involved in remodeling phase through regulating the ECM composition. This facilitates cell migration and neovascularization ${ }^{74}$. Rahmani-Neishaboor et al. ${ }^{71}$ showed that stratifin, a keratinocyte specific 14-3-3 protein, modulated scar formation by inducing the expression of MMP-1 (collagenase) and subsequently reduced the matrix accumulation.

In our study, the effects of ESF, AM and AT-MSCs on relative expression of MMP-1 (collagenase) and MMP-2 (gelatinase) in scar were evaluated. All the wounds exhibited a significant increase in MMP-1 and MMP-2 expression compared to NS ( $\mathrm{P} \leq 0.05)$. In addition, the 
treated wounds (G2-G5) showed a relatively higher MMP-1 and MMP-2 expression in comparison with NTW (G1) $(\mathrm{P} \leq 0.05)$. Figure 8b shows a significant increase of MMP-1 and MMP-2 in AM/ESF treated group compared to AM group. It is reported that TGF- $\beta$ suppress the expression of MMP-1 ${ }^{75}$. In our study, we observed that MMP-1 was up-regulated when the expression of TGF- $\beta$ was decreased. Taken together all the different experimental results suggest that a combination of AM and ESF modulate scar formation by reducing chronic inflammatory response, collagen deposition and also induction of MMPs expression. 


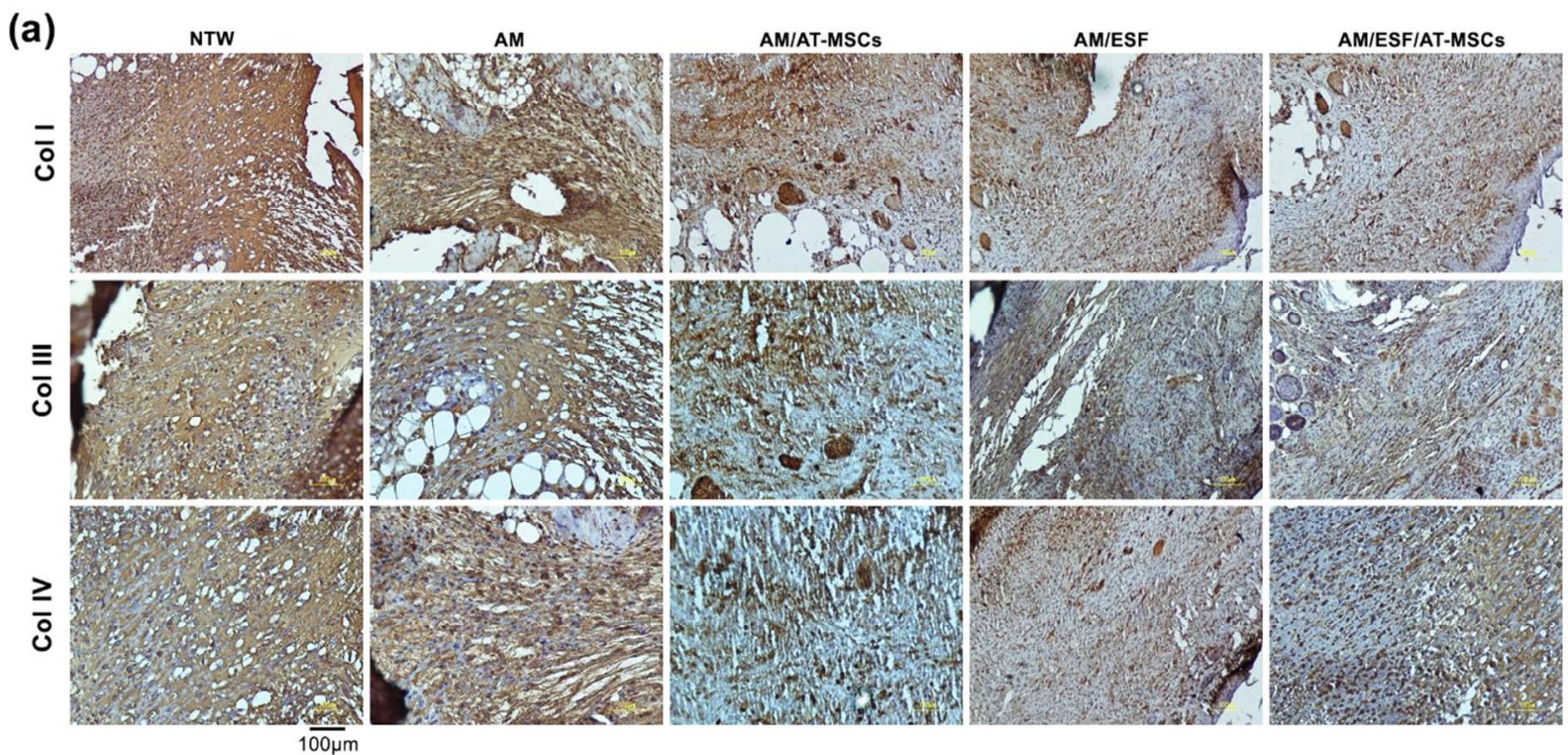

(b)

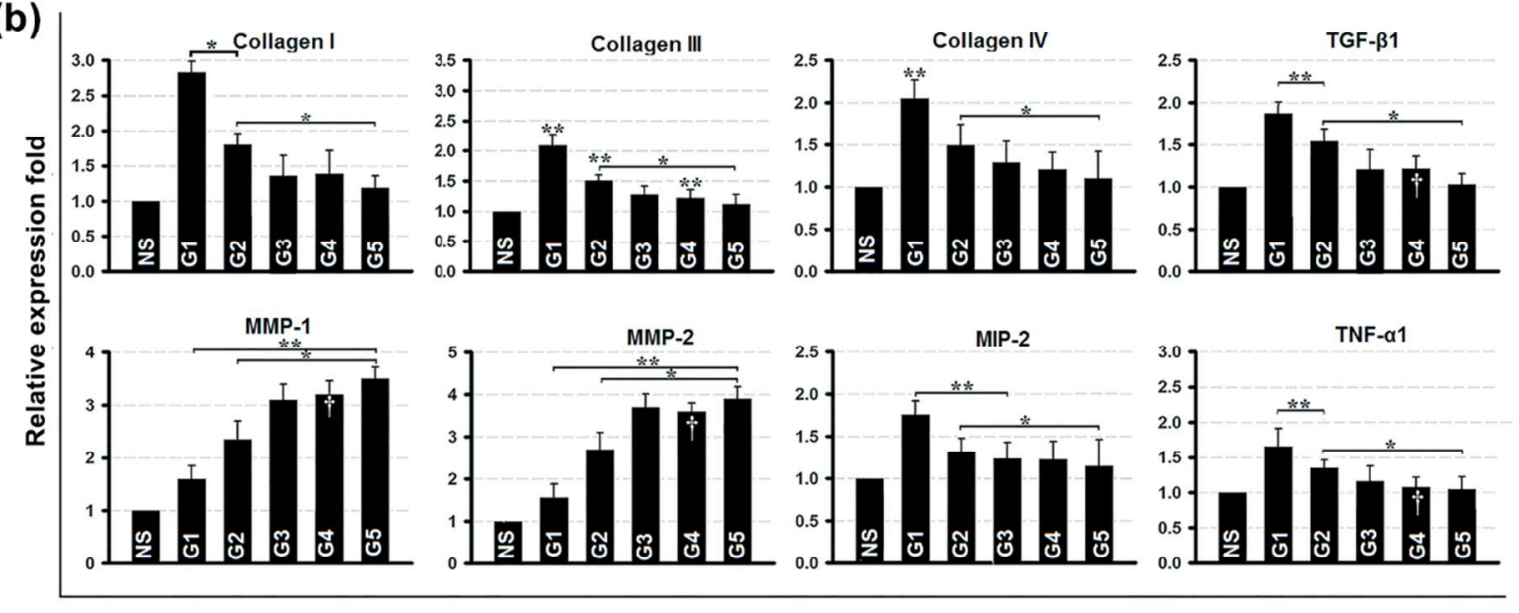

ExperimentalGroups

Figure 8. (a) Deposition of collagen types I, III and IV (COL I, III and IV) in scar tissues of all experimental groups using immunohistochemical staining. Collagens and cell nuclei were stained in brown and dark blue, respectively. (b) The mRNA relative expression of MMP-1, MMP-2, MIP-2, TNF- $\alpha$, Collagen types I, III and IV, and TGF- $\beta 1$ of the scars in all the groups at day 28 of post-operation. NS (Normal skin); G1: NTW (No treated wound) ; G2: AM; G3: AM/AT-MSCs; G4: AM/ESF; G5: AM/ESF/AT-MSCs. * indicates significant difference with 
G1; ** indicates significant difference with NS. $\dagger$ indicates significant difference between G4 and G2.

\section{CONCLUSION}

This study describes the skin burn wound healing potential of 3D bi-layered protein-based artificial skin alone and in combination with mesenchymal stem cells in vivo. Our data suggest that the 3D decellularized human amniotic membrane/electrospun nanofibrous silk fibroin artificial skin in combination with adipose tissue-derived mesenchymal stem cells accelerates significantly scarless wound healing as well as neo-vascularization and early re-epithelialization when implanted to $3^{\text {rd }}$ degree burn wounds in mice. This also indicates that the presence of the electrospun nanofibrous silk fibroin has an important effect on the stem cells differentiation and subsequently facilitating wound healing. Preclinical wound therapy mice model also demonstrates a decreased accumulation of extracellular matrix via collagen deposition, and scar formation. Our fabricated biomaterial composite artificial skin prove both in vitro and in vivo experimentations with wound healing and scar formation and now promises to proceed with clinical investigations.

\section{Supporting Information}

SI Table 1: Recent key researches carried out on scarless healing of skin wounds

SI Table 2: Mouse primers used in RT-PCR analysis

SI Figure 1: H\&Es and Masson's Trichrome (MT) stained sections at day 28 post-operation. 


\begin{abstract}
Authors' contributions
M.G., A.S., and H.G. conceived and designed the study. M.G., S.M.H., H.G. and A.S. performed the experiments. M.G., A.M.S., Z.M., A.M.S., M.D.O. and S.M.H. analyzed the data and interpreted the results. M.G., P.B.M., A.M.U., A.S. and H.G. prepared the manuscript. M.G., J.G.H., A.M.S., A.M.U., S.C.K., and R.L.R reviewed during the preparation of manuscript and revised the manuscript.
\end{abstract}

\title{
Notes
}

The authors declare that there is no conflict of interests regarding the publication of this paper. All authors approved the final version of the manuscript.

\section{Acknowledgement}

We wish to express our appreciation to Prof. Bahram Kazemi and Dr. Nariman Mosaffa for their constructive comments on this manuscript. SC Kundu presently holds an ERA Chair Full Professor position at the 3B's Research Group, University of Minho, Portugal, supported by the European Union Framework Programme for Research and Innovation Horizon 2020 under grant agreement no 668983 - FoReCaST.

\section{Abbreviations}


AM: Amniotic membrane; AM/ESF: Amniotic membrane/electrospun silk fibroin; AT-MSCs: Adipose Tissue-derived mesenchymal stem cells; BC: Blood clot; COL: Collagen; ECs: Epithelial cells; ESF: Electrospun nanofibrous silk fibroin; ET: Ethanol-treated; ETI: Epidermal thickness index; EUT: Ethanol-untreated; HF: Hair Follicle; KCs: Keratinocyte cells; MSCs: Mesenchymal stem cells; MT: Masson's trichrome;NS: Normal skin; NTW: Non-treated wound; PMN: Polymorphonuclear leukocytes; SEI: Scar elevation index; SF: Silk fibroin; G1: controls with $3^{\text {rd }}$ degree burn wound and no implantation (NTW); G2: $3^{\text {rd }}$ degree burn wound implanted with AM alone; G3: $3^{\text {rd }}$ degree burn wound implanted with AM/AT-MSCs construct; G4: $3^{\text {rd }}$ degree burn wound implanted with AM/ESF alone; G5: $3^{\text {rd }}$ degree burn wound implanted with AM/ESF/AT-MSCs construct.

\section{References:}

1. Kowalske, K. J., Burn wound care. Phys. Med. Rehabil. Clin. N. Am. 2011, 22, (2), 213227.

2. Peck, M. D.; Kruger, G. E.; Van Der Merwe, A. E.; Godakumbura, W.; Ahuja, R. B., Burns and fires from non-electric domestic appliances in low and middle income countries: Part I. The scope of the problem. Burns 2008, 34, (3), 303-311.

3. Baranoski, S.; Ayello, E. A., Wound care essentials: Practice principles. Lippincott Williams \& Wilkins: 2nd 2008.

4. WHO, Burns, fact sheet. World Health Organization (http://www.who.int/mediacentre/factsheets/fs365/en/) 2016. 
5. Berman, B.; Maderal, A.; Raphael, B., Keloids and Hypertrophic Scars: Pathophysiology, Classification, and Treatment. Dermatol. Surg. official publication for American Society for Dermatologic Surgery [et al.]. 2016, 43, S3-S18 (doi: 10.1097/DSS.0000000000000819).

6. Lan, Y.; Li, W.; Jiao, Y.; Guo, R.; Zhang, Y.; Xue, W.; Zhang, Y., Therapeutic efficacy of antibiotic-loaded gelatin microsphere/silk fibroin scaffolds in infected full-thickness burns. Acta. Biomater. 2014, 10, (7), 3167-3176.

7. Pham, C.; Greenwood, J.; Cleland, H.; Woodruff, P.; Maddern, G., Bioengineered skin substitutes for the management of burns: a systematic review. Burns 2007, 33, (8), 946-957.

8. Yildirimer, L.; Thanh, N. T.; Seifalian, A. M., Skin regeneration scaffolds: a multimodal bottom-up approach. Trends. Biotechnol. 2012, 30, (12), 638-648.

9. Gholipourmalekabadi, M.; Pal Singh Chauhan, N.; Farhadihosseinabad, B.; Samadikuchaksaraei, A., Human Amniotic Membrane as a Biological Source for Regenerative Medicine. Perinatal Tissue-Derived Stem Cells 2016, Stem Cell Biology and Regenerative Medicine, book series (STEMCELL), 81-105.

10. Wurzer, P.; Keil, H.; Branski, L. K.; Parvizi, D.; Clayton, R. P.; Finnerty, C. C.; Herndon, D. N.; Kamolz, L. P., The use of skin substitutes and burn care-a survey. J. Surg. Res. 2016, 201, (2), 293-298.

11. Anjum, S.; Arora, A.; Alam, M.; Gupta, B., Development of antimicrobial and scar preventive chitosan hydrogel wound dressings. Int. J. Pharm. 2016, 508, (1), 92-101.

12. Gholipourmalekabadi, M.; Samadikuchaksaraei, A.; Seifalian, A. M.; Urbanska, A.; Ghanbarian, H.; Hardy, J. G.; Omrani, M.; Mozafari, M.; Reis, R. L.; Kundu, S. C., Silk fibroin/amniotic membrane 3D bi-layered artificial skin. Biomed. Mater. 2017 (https://doi.org/10.1088/1748-605X/aa999b). 
13. Rostami, A.; Mozafari, M.; Gholipourmalekabadi, M.; Caicedo, H. H.; Lasjerdi, Z.; Sameni, M.; Samadikuchaksaraei, A., Optimization of fluoride-containing bioactive glasses as a novel scolicidal agent adjunct to hydatid surgery. Acta. Trop. 2015, 148, 105-114.

14. Gholipourmalekabadi, M.; Mozafari, M.; Bandehpour, M.; Salehi, M.; Sameni, M.; Caicedo, H. H.; Mehdipour, A.; Hamidabadi, H. G.; Samadikuchaksaraei, A.; Ghanbarian, H., Optimization of nanofibrous silk fibroin scaffold as a delivery system for bone marrow adherent cells: in vitro and in vivo studies. Biotechnol. Appl. Biochem. 2015, 62, (6), 785-794.

15. Garber, J. C.; Barbee, R. W.; Bielitzki, J. T.; Clayton, L.; Donovan, J.; Hendriksen, C.; Kohn, D.; Lipman, N.; Locke, P.; Melcher, J., Guide for the care and use of laboratory animals. The National Academic Press, Washington DC 2011, 8, 220.

16. Shahabi, S.; Hashemi, M.; Hassan, Z. M.; Javan, M.; Bathaie, S. Z.; Toraihi, T.; Zakeri, Z.; Ilkhanizadeh, B.; Jazani, N. H., The effect of post-burn local hyperthermia on the reducing burn injury: the possible role of opioids. Int. J. Hyperthermia. 2006, 22, (5), 421-431.

17. Weavers, H.; Liepe, J.; Sim, A.; Wood, W.; Martin, P.; Stumpf, M. P., Systems analysis of the dynamic inflammatory response to tissue damage reveals spatiotemporal properties of the wound attractant gradient. Curr. Biol. 2016, 26, (15), 1975-1989.

18. Jeong, L.; Kim, M. H.; Jung, J.-Y.; Min, B. M.; Park, W. H., Effect of silk fibroin nanofibers containing silver sulfadiazine on wound healing. Int. J. Nanomed. 2014, 9, 5277.

19. Lorden, E. R.; Miller, K. J.; Bashirov, L.; Ibrahim, M. M.; Hammett, E.; Jung, Y.; Medina, M. A.; Rastegarpour, A.; Selim, M. A.; Leong, K. W., Mitigation of hypertrophic scar contraction via an elastomeric biodegradable scaffold. Biomaterials 2015, 43, 61-70. 
20. Shan, Y.-H.; Peng, L.-H.; Liu, X.; Chen, X.; Xiong, J.; Gao, J.-Q., Silk fibroin/gelatin electrospun nanofibrous dressing functionalized with astragaloside IV induces healing and antiscar effects on burn wound. Int. J. Pharm. 2015, 479, (2), 291-301.

21. Cheng, L.; Sun, X.; Zhao, X.; Wang, L.; Yu, J.; Pan, G.; Li, B.; Yang, H.; Zhang, Y.; Cui, W., Surface biofunctional drug-loaded electrospun fibrous scaffolds for comprehensive repairing hypertrophic scars. Biomaterials 2016, 83, 169-181.

22. Rahmani-Neishaboor, E.; Yau, F. M. k.; Jalili, R.; Kilani, R. T.; Ghahary, A., Improvement of hypertrophic scarring by using topical anti-fibrogenic/anti-inflammatory factors in a rabbit ear model. Wound. Repair. Regen. 2010, 18, (4), 401-408.

23. Zonari, A.; Martins, T. M.; Paula, A. C. C.; Boeloni, J. N.; Novikoff, S.; Marques, A. P.; Correlo, V. M.; Reis, R. L.; Goes, A. M., Polyhydroxybutyrate-co-hydroxyvalerate structures loaded with adipose stem cells promote skin healing with reduced scarring. Acta. biomater. 2015, 17, 170-181.

24. Yan, S.; Zhang, Q.; Wang, J.; Liu, Y.; Lu, S.; Li, M.; Kaplan, D. L., Silk fibroin/chondroitin sulfate/hyaluronic acid ternary scaffolds for dermal tissue reconstruction. Acta. biomater. 2013, 9, (6), 6771-6782.

25. Mohsenzadegan, M.; Madjd, Z.; Asgari, M.; Abolhasani, M.; Shekarabi, M.; Taeb, J.; Shariftabrizi, A., Reduced expression of NGEP is associated with high-grade prostate cancers: a tissue microarray analysis. Cancer Immunol Immunother. 2013, 62, (10), 1609-1618.

26. Vasconcelos, A.; Gomes, A. C.; Cavaco-Paulo, A., Novel silk fibroin/elastin wound dressings. Acta. biomater. 2012, 8, (8), 3049-3060.

27. Wu, Y.; Chen, L.; Scott, P. G.; Tredget, E. E., Mesenchymal stem cells enhance wound healing through differentiation and angiogenesis. Stem cells 2007, 25, (10), 2648-2659. 
28. Okabayashi, R.; Nakamura, M.; Okabayashi, T.; Tanaka, Y.; Nagai, A.; Yamashita, K., Efficacy of polarized hydroxyapatite and silk fibroin composite dressing gel on epidermal recovery from full-thickness skin wounds. J. Biomed. Mater. Res. B: Appl. Biomater. 2009, 90, (2), 641-646.

29. Wendt, H.; Hillmer, A.; Reimers, K.; Kuhbier, J. W.; Schäfer-Nolte, F.; Allmeling, C.; Kasper, C.; Vogt, P. M., Artificial skin-culturing of different skin cell lines for generating an artificial skin substitute on cross-weaved spider silk fibres. PloS one 2011, 6, (7), e21833.

30. Altman, A. M.; Yan, Y.; Matthias, N.; Bai, X.; Rios, C.; Mathur, A. B.; Song, Y. H.; Alt, E. U., IFATS Collection: Human Adipose-Derived Stem Cells Seeded on a Silk FibroinChitosan Scaffold Enhance Wound Repair in a Murine Soft Tissue Injury Model. Stem Cells 2009, $27,(1), 250-258$.

31. Shefa, A. A.; Amirian, J.; Kang, H. J.; Bae, S. H.; Jung, H.-I.; Choi, H.-j.; Lee, S. Y.; Lee, B.-T., In vitro and in vivo evaluation of effectiveness of a novel TEMPO-oxidized cellulose nanofiber-silk fibroin scaffold in wound healing. Carbohydr. Polym. 2017, 177, 284-296.

32. Ito, M.; Cotsarelis, G., Is the hair follicle necessary for normal wound healing? $J$. Investig. Dermatol. 2008, 128, (5), 1059-1061.

33. Kanokpanont, S.; Damrongsakkul, S.; Ratanavaraporn, J.; Aramwit, P., Physico-chemical properties and efficacy of silk fibroin fabric coated with different waxes as wound dressing. Int. J. Biol. Macromol. 2013, 55, 88-97.

34. Sabapathy, V.; Sundaram, B.; Sreelakshmi, V.; Mankuzhy, P.; Kumar, S., Human Wharton's jelly mesenchymal stem cells plasticity augments scar-free skin wound healing with hair growth. PloS one 2014, 9, (4), e93726. 
35. Yang, M.-C.; Wang, S.-S.; Chou, N.-K.; Chi, N.-H.; Huang, Y.-Y.; Chang, Y.-L.; Shieh, M.-J.; Chung, T.-W., The cardiomyogenic differentiation of rat mesenchymal stem cells on silk fibroin-polysaccharide cardiac patches in vitro. Biomaterials 2009, 30, (22), 3757-3765.

36. Fan, H.; Liu, H.; Toh, S. L.; Goh, J. C., Enhanced differentiation of mesenchymal stem cells co-cultured with ligament fibroblasts on gelatin/silk fibroin hybrid scaffold. Biomaterials 2008, 29, (8), 1017-1027.

37. Schneider, A.; Wang, X.; Kaplan, D.; Garlick, J.; Egles, C., Biofunctionalized electrospun silk mats as a topical bioactive dressing for accelerated wound healing. Acta. Biomater. 2009, 5, (7), 2570-2578.

38. Bhatia, M.; Pereira, M.; Rana, H.; Stout, B.; Lewis, C.; Abramson, S.; Labazzo, K.; Ray, C.; Liu, Q.; Hofgartner, W., The mechanism of cell interaction and response on decellularized human amniotic membrane: implications in wound healing. Wounds: a compendium of clinical research and practice 2007, 19, (8), 207-217.

39. Woo, H.; Kim, M.; Kweon, O.; Kim, D.; Nam, T.; Kim, J., Effects of amniotic membrane on epithelial wound healing and stromal remodelling after excimer laser keratectomy in rabbit cornea. Br. J. Ophthalmol. 2001, 85, (3), 345-349.

40. Locksley, R. M.; Killeen, N.; Lenardo, M. J., The TNF and TNF receptor superfamilies: integrating mammalian biology. Cell 2001, 104, (4), 487-501.

41. Theiss, A. L.; Simmons, J. G.; Jobin, C.; Lund, P. K., Tumor necrosis factor (TNF) $\alpha$ increases collagen accumulation and proliferation in intestinal myofibroblasts via TNF receptor 2. J. Biol. Chem. 2005, 280, (43), 36099-36109.

42. Spiekman, M.; Przybyt, E.; Plantinga, J. A.; Gibbs, S.; van der Lei, B.; Harmsen, M. C., Adipose Tissue-Derived Stromal Cells Inhibit TGF- $\beta 1$-Induced Differentiation of Human 
Dermal Fibroblasts and Keloid Scar-Derived Fibroblasts in a Paracrine Fashion. Plast. Reconstr. Surg. 2014, 134, (4), 699-712.

43. Velnar, T.; Bailey, T.; Smrkolj, V., The wound healing process: an overview of the cellular and molecular mechanisms. J. Int. Med. Res. 2009, 37, (5), 1528-1542.

44. Price, R.; Anthony, E.; Myers, S.; Navsaria, H., Tissue engineering for skin transplantation. Biomed. Eng. 2008, 2, 507-532.

45. Clark, R. A.; Ghosh, K.; Tonnesen, M. G., Tissue engineering for cutaneous wounds. J. Investig. Dermatol. 2007, 127, (5), 1018-1029.

46. Guo, S.; DiPietro, L. A., Factors affecting wound healing. J. Dent. Res. 2010, 89, (3), $219-229$.

47. Kondo, T.; Ishida, Y., Molecular pathology of wound healing. Forensic Sci. Int. 2010, 203, (1), 93-98.

48. Tammela, T.; Enholm, B.; Alitalo, K.; Paavonen, K., The biology of vascular endothelial growth factors. Cardiovasc. Res. 2005, 65, (3), 550-563.

49. Szczepkowska, A.; Lagaraine, C.; Robert, V.; Młynarczuk, J.; Dufourny, L.; Thiéry, J.C.; Skipor, J., PCB153 (2, 2', 4, 4', 5, 5'-hexachlorobiphenyl) differentially affects the VEGF/VEGFR system depending on photoperiod in the ovine choroid plexus. Ecotoxicol. Environ. Saf. 2016, 124, 303-308.

50. Li, S.; Li, L.; Guo, C.; Qin, H.; Yu, X., A promising wound dressing material with excellent cytocompatibility and proangiogenesis action for wound healing: Strontium loaded Silk fibroin/Sodium alginate (SF/SA) blend films. Int. J. Biol. Macromol. 2017, 104, 969-978. 
51. Stojkovic, P.; Lako, M.; Stewart, R.; Przyborski, S.; Armstrong, L.; Evans, J.; Murdoch, A.; Strachan, T.; Stojkovic, M., An autogeneic feeder cell system that efficiently supports growth of undifferentiated human embryonic stem cells. Stem Cells 2005, 23, (3), 306-314.

52. Shibuya, M., Vascular endothelial growth factor and its receptor system: physiological functions in angiogenesis and pathological roles in various diseases. J. Biochem. 2013, 153, (1), 13-19.

53. Seyfarth, H.; Sack, U.; Gessner, C.; Wirtz, H., [Angiogenin, bFGF and VEGF: angiogenic markers in breath condensate of patients with pulmonary hypertension]. Pneumologie (Stuttgart, Germany) 2015, 69, (4), 207-211.

54. Barrientos, S.; Stojadinovic, O.; Golinko, M. S.; Brem, H.; Tomic-Canic, M., Growth factors and cytokines in wound healing. Wound. Repair. Regen. 2008, 16, (5), 585-601.

55. Ferretti, C.; Vozzi, G.; Falconi, M.; Orciani, M.; Gesi, M.; Di Primio, R.; MattioliBelmonte, M., Role of IGF1 and IGF1/VEGF on human mesenchymal stromal cells in bone healing: two sources and two fates. Tissue. Eng. Part. A. 2014, 20, (17-18), 2473-2482.

56. Strand, V.; Kavanaugh, A., The role of interleukin-1 in bone resorption in rheumatoid arthritis. Rheumatology-Oxford 2004, 43, (3), iii10.

57. Bai, L.; Wu, D.; Xu, J.; Liu, H.; Xie, M.; Guan, G.; Sun, Z.; Tan, X., On model of angiogenesis and the mechanism in porous silk fibroin films. J. Mater. Sci. Mater. Med. 2011, 22, (4), 927-933.

58. Zhan, K. H.; Bai, L.; Guan, G. P.; Dai, H. Q., Characterization of angiogenesis during skin wound repair by porous silk fibroin film. Adv. Mater. Res. 2011; Trans Tech Publ: 2011; pp 181-185 (doi: 10.4028/www.scientific.net/AMR.175-176.181). 
59. Unger, R.; Peters, K.; Wolf, M.; Motta, A.; Migliaresi, C.; Kirkpatrick, C., Endothelialization of a non-woven silk fibroin net for use in tissue engineering: growth and gene regulation of human endothelial cells. Biomaterials 2004, 25, (21), 5137-5146.

60. Fuchs, S.; Motta, A.; Migliaresi, C.; Kirkpatrick, C. J., Outgrowth endothelial cells isolated and expanded from human peripheral blood progenitor cells as a potential source of autologous cells for endothelialization of silk fibroin biomaterials. Biomaterials 2006, 27, (31), 5399-5408.

61. Gil, E. S.; Panilaitis, B.; Bellas, E.; Kaplan, D. L., Functionalized silk biomaterials for wound healing. Adv. Healthc. Mater. 2013, 2, (1), 206-217.

62. Han, H.; Ning, H.; Liu, S.; Lu, Q.; Fan, Z.; Lu, H.; Lu, G.; Kaplan, D. L., Silk Biomaterials with Vascularization Capacity. Adv. Funct. Mater. 2016, 26, (3), 421-432.

63. Foubert, P.; Gonzalez, A. D.; Teodosescu, S.; Berard, F.; Doyle-Eisele, M.; Yekkala, K.; Tenenhaus, M.; Fraser, J. K., Adipose-Derived Regenerative Cell Therapy for Burn Wound Healing: A Comparison of Two Delivery Methods. Adv. Wound .Care. 2015, 5, (7), 288-298.

64. Shingyochi, Y.; Orbay, H.; Mizuno, H., Adipose-derived stem cells for wound repair and regeneration. Expert. Opin. Biol. Ther. 2015, 15, (9), 1285-1292.

65. Chiang, R. S.; Borovikova, A. A.; King, K.; Banyard, D. A.; Lalezari, S.; Toranto, J. D.; Paydar, K. Z.; Wirth, G. A.; Evans, G. R.; Widgerow, A. D., Current concepts related to hypertrophic scarring in burn injuries. Wound. Repair. Regen. 2016, 24, (3), 466-477.

66. Gauglitz, G. G.; Korting, H. C.; Pavicic, T.; Ruzicka, T.; Jeschke, M. G., Hypertrophic scarring and keloids: pathomechanisms and current and emerging treatment strategies. Mol. Med. 2011, 17, (1-2), 113-125. 
67. Acharya, C.; Hinz, B.; Kundu, S. C., The effect of lactose-conjugated silk biomaterials on the development of fibrogenic fibroblasts. Biomaterials 2008, 29, (35), 4665-4675.

68. Du, L.; Lv, R.; Yang, X.; Cheng, S.; Ma, T.; Xu, J., Hypoxic conditioned medium of placenta-derived mesenchymal stem cells protects against scar formation. Life. sci. 2016, 149, $51-57$.

69. Cheng, W.; Yan-hua, R.; Fang-gang, N.; Guo-an, Z., The content and ratio of type I and III collagen in skin differ with age and injury. Afr. J. Biotechnol. 2011, 10, (13), 2524-2529.

70. Butzelaar, L.; Ulrich, M.; van der Molen, A. M.; Niessen, F.; Beelen, R., Currently known risk factors for hypertrophic skin scarring: A review. J. Plast. Reconstr. Aesthet. Surg. 2016, 69, (2), 163-169.

71. Rahmani-Neishaboor, E.; Jackson, J.; Burt, H.; Ghahary, A., Composite hydrogel formulations of stratifin to control MMP-1 expression in dermal fibroblasts. Pharm. Res. 2009, 26, (8), 2002-2014.

72. Falomo, M. E.; Ferroni, L.; Tocco, I.; Gardin, C.; Zavan, B., Immunomodulatory Role of Adipose-Derived Stem Cells on Equine Endometriosis. BioMed. Res. Int. 2015, 2015 (doi: http://dx.doi.org/10.1155/2015/141485).

73. Ong, W. K.; Sugii, S., Adipose-derived stem cells: fatty potentials for therapy. Int. J. Biochem. Cell Biol. 2013, 45, (6), 1083-1086.

74. Scharf, B.; Clement, C. C.; Yodmuang, S.; Urbanska, A. M.; Suadicani, S. O.; Aphkhazava, D.; Thi, M. M.; Perino, G.; Hardin, J. A.; Cobelli, N., Age-related carbonylation of fibrocartilage structural proteins drives tissue degenerative modification. Chem. Biol. 2013, 20, (7), 922-934. 
75. Wang, J.; Jiao, H.; Stewart, T. L.; Shankowsky, H. A.; Scott, P. G.; Tredget, E. E., Increased TGF- $\beta$-producing CD4+ $\mathrm{T}$ lymphocytes in postburn patients and their potential interaction with dermal fibroblasts in hypertrophic scarring. Wound. Repair.Regen. 2007, 15, (4), $530-539$.

TOC

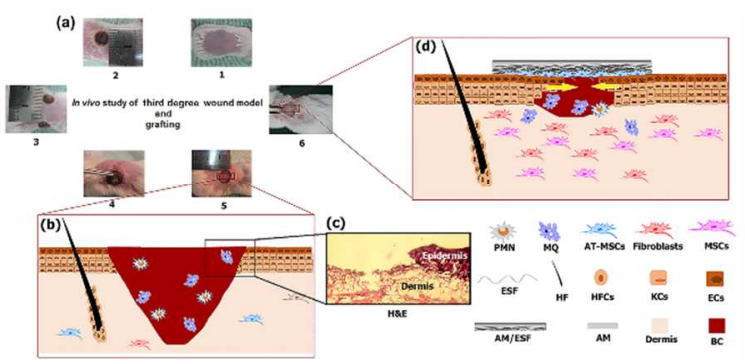

